\title{
Chemical analysis of NGC 6528: one of the most metal-rich bulge globular clusters
}

\author{
C. Muñoz ${ }^{1}$, D. Geisler ${ }^{1,5,6}$, S. Villanova ${ }^{1}$, I. Saviane ${ }^{2}$, C. C. Cortés ${ }^{1}$, B. Dias ${ }^{2}$, R. E. Cohen ${ }^{3}$, \\ F. Mauro ${ }^{4}$, and C. Moni Bidin ${ }^{4}$
}

1 Departamento de Astronomía, Casilla 160-C, Universidad de Concepción, Concepción, Chile e-mail: cesarmunoz@astro-udec.cl

2 European Southern Observatory, Casilla 19001 Santiago, Chile

3 Space Telescope Science Institute, 3700 San Martin Drive, Baltimore, MD 21218, USA

${ }^{4}$ Instituto de Astronomía, Universidad Católica del Norte, Av. Angamos 0610, Antofagasta, Chile

5 Instituto de Investigación Multidisciplinario en Ciencia y Tecnología, Universidad de La Serena. Avenida Raúl Bitrán s/n, La Serena, Chile

${ }^{6}$ Departamento de Física y Astronomía, Facultad de Ciencias, Universidad de La Serena. Av. Juan Cisternas, 1200 La Serena, Chile

Received 5 May 2018 / Accepted 21 August 2018

\begin{abstract}
Context. The bulge globular clusters (GCs) are key tracers of the bulge, a central and ancient component of our Galaxy. It is essential to understand their formation and evolution to study that of the bulge, as well as their relationship with the other Galactic GC systems (halo and disk GCs). High-resolution spectroscopy is a powerful tool for such studies, allowing us to obtain a detailed chemical characterization and kinematics of the clusters and to compare their chemical patterns with those of their halo and disk counterparts. Aims. Our main goals are to obtain detailed abundances for a sample of seven red giant members of NGC 6528 in order to characterize their chemical composition and study the relationship of this GC with the bulge, and with other bulge, halo, and disk GCs. Moreover, we analyze this cluster's behavior associated with the multiple-populations phenomenon.

Methods. We obtained the stellar parameters and chemical abundances of light elements ( $\mathrm{Na}, \mathrm{Al})$, iron-peak elements $(\mathrm{V}, \mathrm{Cr}, \mathrm{Mn}$, $\mathrm{Fe}, \mathrm{Co}, \mathrm{Ni}, \mathrm{Cu}), \alpha$-elements $(\mathrm{O}, \mathrm{Mg}, \mathrm{Si}, \mathrm{Ca}, \mathrm{Ti})$ and heavy elements $(\mathrm{Zr}, \mathrm{Ba}, \mathrm{Eu})$ in seven red giant members of NGC 6528 using high-resolution spectroscopy from FLAMES-UVES.

Results. In six stars of our sample we obtained a mean iron content of $[\mathrm{Fe} / \mathrm{H}]=-0.14 \pm 0.03$ dex, in good agreement with other studies. We found no significant internal iron spread. We detected one candidate variable star, which was excluded from the mean in iron content, and derived a metallicity in this star of $[\mathrm{Fe} / \mathrm{H}]=-0.55 \pm 0.04$ dex. Moreover, we found no extended O-Na anticorrelation but instead only an intrinsic Na spread. In addition, NGC 6528 does not exhibit a Mg-Al anticorrelation, and no significant spread in either $\mathrm{Mg}$ or $\mathrm{Al}$. The $\alpha$ and iron-peak elements show good agreement with the bulge field star trend. The heavy elements are slightly dominated by the $r$-process. The chemical analysis suggests an origin and evolution similar to that of typical old Galactic bulge field stars. Finally, we find remarkable agreement in the chemical patterns of NGC 6528 and another bulge GC, NGC 6553, suggesting a similar origin and evolution.
\end{abstract}

Key words. stars: abundances - globular clusters: individual: NGC 6528 - globular clusters: general - Galaxy: bulge stars: atmospheres

\section{Introduction}

The detailed study of each main component of our Galaxy is critical to understanding their formation and evolution, as well as that of the Galaxy as a whole. This is graphically illustrated by the many current and new surveys that seek to extend our Galactic knowledge, such as: the VVV survey (Minniti et al. 2010), the Gaia-ESO survey (Gilmore et al. 2012), SDSS-IV (Blanton et al. 2017), and the Gaia mission.

One of the main components of our Galaxy is the bulge, which, being likely the oldest Galactic component (e.g., Cescutti et al. 2018), provides us with invaluable information about the origin and subsequent evolution of our Galaxy. One of the key tracers of the bulge is the large number of GCs concentrated there, as first famously realized by Shapley, which we now recognize form an independent GC system from that of the halo (Minniti 1995). Despite the observational difficulties associated with detailed investigations of such GCs, particularly crowding in this dense central region of the Galaxy and strong and variable reddening, their importance is such that more and more studies are exploring these fundamental objects.

In general, the GCs that have been studied in the more accessible halo and disk have been characterized by inhomogeneities in their light-element content (C, N, O, Na, Mg and/or Al), most prominently appearing as $\mathrm{O}-\mathrm{Na}$ and $\mathrm{Mg}-\mathrm{Al}$ anticorrelations. In fact, the O-Na anticorrelation has been found in almost all Galactic GCs (Carretta et al. 2009b) and has even been suggested as a defining characteristic of GCs (Carretta et al. 2009a,b, 2010b), with Ruprecht 106 (Villanova et al. 2013) being the most likely exception.

These chemical patterns must be due to the self-enrichment that GCs suffer in the early stages of their formation, allowing the formation of multiple generations of stars within the GC (Gratton et al. 2004). Suggested polluters include intermediatemass asymptotic giant branch (AGB) stars (D'Antona et al. 2002, 2016), fast rotating massive main sequence (MS) stars 
(Decressin et al. 2007; Krause et al. 2013) and massive binaries (de Mink et al. 2010; Izzard et al. 2013).

The spread in iron content is another peculiarity that has been observed in a smaller fraction of GCs, although in some cases this needs to be corroborated with more detailed studies, as in the case of NGC 3201 (Simmerer et al. 2013; Muñoz et al. 2013; Mucciarelli et al. 2015). In general, some massive GCs in particular display iron variations, most notably Omega Cen (Johnson et al. 2008; Marino et al. 2011a), M22 (Marino et al. 2011b; Da Costa et al. 2009), M54 (Carretta et al. 2010a) and Terzan 5 (Origlia et al. 2011).

The study of bulge GCs, although lagging behind until now because of observational issues, has recently seen increasing efforts, allowing important improvements in our previously very scanty knowledge of these key objects. In our first paper on bulge GCs, focusing on NGC 6440 (Muñoz et al. 2017), we found that the evolution of some bulge GCs is not as similar to that of the halo GCs as often postulated. Additionally, the bulge GCs show great agreement with the overall chemistry of bulge field stars (Muñoz et al. 2017). However, the main problems limiting the study of bulge GCs remain the large and variable extinction and high crowding.

In this second paper, we have studied the bulge GC NGC 6528. It is located at $\left(l=1^{\circ}, b=-4^{\circ}\right)$, at a distance of $0.6 \mathrm{Kpc}$ from the Galactic center with a nominal reddening of $E(B-V)=$ 0.54 (Harris 1996). It is a rather well-studied bulge GC, lying in Baade's window and very near another bulge GC, NGC 6522. NGC 6528 has been studied by several authors with different instruments and techniques. Carretta et al. (2001) studied four red horizontal branch (RHB) stars using the high-resolution spectrograph HIRES from the Keck telescope. Origlia et al. (2005) studied four red giant branch (RGB) stars using the Near-Infrared Spectrometer Spectrograph (NIRSPEC) at Keck II, measuring the chemical abundances for six different elements. Also, Schiavon et al. (2017) reported chemical abundances for six elements, which were obtained by Sloan Digital Sky Survey (SDSS)-III. Saviane et al. (2012) studied this cluster using the calcium triplet technique, Lagioia et al. (2014) analyzed this cluster using optical photometry from HST, Cohen et al. (2017) used near-infrared photometry, Dias et al. (2015) studied a large sample of GCs, among them NGC 6528, with low-resolution spectroscopy, and Zoccali et al. (2004) used high-resolution spectroscopy and found overall metallicity $Z \approx Z_{\odot}$. It is particularly interesting that Mauro et al. (2014) found a possible iron spread in this GC based on the low-resolution Ca triplet spectra of seven stars obtained by Saviane et al. (2012). We have made a detailed chemical characterization of this GC, measuring the abundance of 18 elements including light elements, $\alpha$ elements, iron-peak and heavy elements, yielding a much more detailed picture of the chemical evolution of this GC than previously available.

In Sect. 2 we describe the observations and data reduction, in Sect. 3 we explain the methodology we used to calculate atmospheric parameters, errors, and chemical abundances. In Sect. 4 we present our results concerning the variability in one star of our sample and abundances for iron-peak elements, $\alpha$ elements, $\mathrm{Na}-\mathrm{O}$ anticorrelation, $\mathrm{Mg}-\mathrm{Al}$ relations and heavy elements. Finally, in Sect. 5 our conclusions are presented.

\section{Observations and data reduction}

We observed red giants towards NGC 6528 with the fiberfed multiobject FLAMES spectrograph mounted at the ESO VLT/UT2 telescope in Cerro Paranal (Chile; ESO program ID

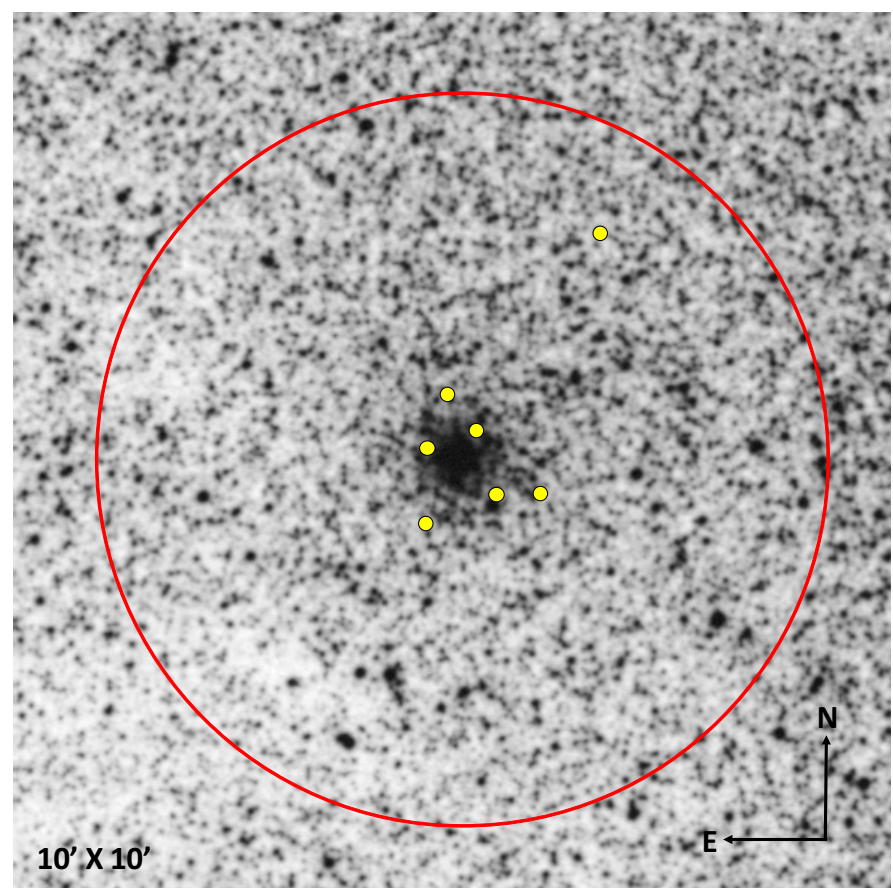

Fig. 1. Distribution of the stars observed in NGC 6528 (yellow filled circles). The red circle is the tidal radius (Harris 1996).

093.D-0286, PI S. Villanova). Here we analyze the seven stars observed with the blue and red arms of the high-resolution spectrograph UVES. FLAMES-UVES data have a spectral resolution of about $R \simeq 47000$. The data were taken with central wavelength $580 \mathrm{~nm}$, which covers the wavelength range $476-684 \mathrm{~nm}$. Our signal-to-noise ratio $(\mathrm{S} / \mathrm{N})$ is approximately 30 at $650 \mathrm{~nm}$.

Six of the seven targets observed with FLAMES at UVES came from the membership list of NGC 6528 previously published in Saviane et al. (2012) and Mauro et al. (2014) using FORS2 and VVV. Star \#1 is not part of any previous study, but was selected as a likely member based on its position in the color magnitude diagram (CMD). Our subsequent analysis shows that all seven stars are indeed members taking into account the radial velocity, metallicity, CMD position, and detailed chemical patterns. Their spatial distribution is shown in Fig. 1.

All the stars of our sample belong to the upper RGB, as can be clearly seen in the CMD of the cluster (Fig. 2). Star \#5 will be discussed in Sect. 4.1.

Data reduction was performed using the ESO CPL based FLAMES/UVES Pipeline version 5.3.0 ${ }^{1}$ for extracting the individual fiber spectra. Data reduction includes bias subtraction, flat-field correction, wavelength calibration, and spectral rectification.

We subtracted the sky using the Sarith package in IRAF and measured radial velocities using the FXCOR package in IRAF and a synthetic spectrum as a template. The mean heliocentric radial velocity for our seven targets is $210.8 \pm 2.5 \mathrm{~km} \mathrm{~s}^{-1}$. Our velocity dispersion is $6.7 \mathrm{~km} \mathrm{~s}^{-1}$.

Our mean radial velocity is compatible with the values in the literature: Saviane et al. (2012) with four stars found a value of $205 \pm 2 \mathrm{~km} \mathrm{~s}^{-1}$, Harris (1996) quotes a value of $206.6 \pm 1.4 \mathrm{~km} \mathrm{~s}^{-1}$, Carretta et al. (2001) found a value of $210 \mathrm{~km} \mathrm{~s}^{-1}$, Schiavon et al. (2017) reported a mean radial velocity of $213 \mathrm{~km} \mathrm{~s}^{-1}$ for two stars and Origlia et al. (2005) found

http://wWw . eso.org/sci/software/pipelines/ 
Table 1. Parameters of the observed stars.

\begin{tabular}{ccccccccccc}
\hline \hline ID & $\begin{array}{c}\text { RA } \\
(\mathrm{h}: \mathrm{m}: \mathrm{s})\end{array}$ & $\begin{array}{c}\mathrm{Dec} \\
\left({ }^{\circ} !^{\prime \prime}\right)\end{array}$ & $\begin{array}{c}J \\
(\mathrm{mag})\end{array}$ & $\begin{array}{c}H \\
(\mathrm{mag})\end{array}$ & $\begin{array}{c}K_{s} \\
(\mathrm{mag})\end{array}$ & $\begin{array}{c}\mathrm{RV}_{H} \\
\left(\mathrm{~km} \mathrm{~s}^{-1}\right)\end{array}$ & $\begin{array}{c}T_{\text {eff }} \\
(\mathrm{K})\end{array}$ & $\begin{array}{c}\log (\mathrm{g}) \\
{[\mathrm{Fe} / \mathrm{H}]} \\
\mathrm{dex}\end{array}$ & $\begin{array}{c}v_{t} \\
\left(\mathrm{~km} \mathrm{~s} \mathrm{~s}^{-1}\right)\end{array}$ \\
\hline 1 & $18: 04: 42.16$ & $-30: 00: 49.70$ & 12.62 & 11.72 & 11.49 & $198.93 \pm 0.21$ & 4133 & 1.67 & -0.16 & 1.14 \\
2 & $18: 04: 51.32$ & $-30: 04: 05.68$ & 12.76 & 11.92 & 11.67 & $209.91 \pm 0.14$ & 4357 & 2.00 & -0.12 & 1.43 \\
3 & $18: 04: 47.76$ & $-30: 03: 47.05$ & 12.71 & 11.82 & 11.56 & $215.30 \pm 0.16$ & 4232 & 2.03 & -0.24 & 1.52 \\
4 & $18: 04: 50.13$ & $-30: 02: 38.05$ & 12.88 & 12.02 & 11.81 & $216.08 \pm 0.20$ & 4369 & 2.14 & -0.11 & 1.42 \\
5 & $18: 04: 45.38$ & $-30: 03: 46.94$ & 11.87 & 10.95 & 10.68 & $209.70 \pm 0.23$ & 4118 & 1.58 & -0.55 & 1.79 \\
6 & $18: 04: 51.17$ & $-30: 03: 14.95$ & 12.32 & 11.43 & 11.15 & $218.71 \pm 0.19$ & 4120 & 1.39 & -0.20 & 1.27 \\
7 & $18: 04: 48.75$ & $-30: 03: 01.88$ & 13.05 & 12.23 & 12.00 & $206.91 \pm 0.18$ & 4299 & 1.86 & -0.03 & 1.12 \\
\hline
\end{tabular}

Notes. The $J, H, K_{s}$ magnitudes are de-reddened PSF photometry of VVV (Mauro et al. 2014; Cohen et al. 2017).

Table 2. Iron abundances from previous studies.

\begin{tabular}{ccccc}
\hline \hline ID & {$[\mathrm{Fe} / \mathrm{H}]_{\text {this_work }}$} & {$[\mathrm{Fe} / \mathrm{H}]_{\mathrm{S} 12}$} & {$[\mathrm{Fe} / \mathrm{H}]_{\mathrm{M} 14}$} & {$[\mathrm{Fe} / \mathrm{H}]_{\mathrm{L} 17}$} \\
\hline 1 & $-0.16 \pm 0.06$ & - & - & - \\
2 & $-0.12 \pm 0.06$ & - & $-0.15 \pm 0.14$ & - \\
3 & $-0.24 \pm 0.06$ & - & $-0.27 \pm 0.14$ & $-0.07 \pm 0.12$ \\
4 & $-0.11 \pm 0.06$ & $-0.38 \pm 0.16$ & $-0.50 \pm 0.14$ & - \\
5 & $-0.55 \pm 0.06$ & - & $-0.17 \pm 0.14$ & $-0.15 \pm 0.09$ \\
6 & $-0.20 \pm 0.06$ & $-0.22 \pm 0.16$ & $-0.44 \pm 0.14$ & $-0.17 \pm 0.12$ \\
7 & $-0.03 \pm 0.06$ & $-0.57 \pm 0.16$ & $-0.64 \pm 0.14$ & - \\
\hline
\end{tabular}

Notes. S12: Saviane et al. (2012); M14: Mauro et al. (2014); L17: Liu et al. (2017).

a value of $210 \mathrm{~km} \mathrm{~s}^{-1}$ in a sample of four RGB stars. Table 1 lists the stellar parameters of our sample: identity (ID), the J2000 coordinates (RA and Dec), $J, H, K_{s}$ magnitudes from VVV PSF photometry (de-reddened) calibrated on the system of 2MASS (Mauro et al. 2014; Cohen et al. 2017), heliocentric radial velocity, $T_{\text {eff }}, \log (\mathrm{g})$, metallicity and micro-turbulent velocity $\left(v_{t}\right)$. Moreover, Table 2 shows the metallicity values from Saviane et al. (2012), Mauro et al. (2014) and Liu et al. (2017).

\section{Atmospheric parameters and abundances}

We analyzed our sample of seven members of NGC 6528 using the local thermodynamic equilibrium (LTE) program MOOG (Sneden 1973). The procedure used in this paper to calculate the atmospheric parameters is the same that was described in our previous study of NGC 6440 (Muñoz et al. 2017). In Fig. 4 we show the good agreement between our stellar parameters and an isochrone of similar metallicity as we derive $([\mathrm{Fe} / \mathrm{H}]=-0.14$ dex) and an age of 11 Gyr (Dotter et al. 2008).

Although NGC 6528 lies towards the bulge, its location in Baade's Window means the extinction is not as high as for other nearby regions with similar Galactic coordinates. The mean color excess quoted by Harris (1996) is $E(B-V)=0.54$ and Momany et al. (2003) found a very similar value of $E(B-V)=$ 0.55 . Moreover, like the majority of bulge GCs, it is assumed to have differential reddening and certainly high crowding being in Baade's Window, which complicates the identification of member stars. We determined the stellar parameters directly from the spectra, so our measurement of abundances is not affected by the effects of reddening.

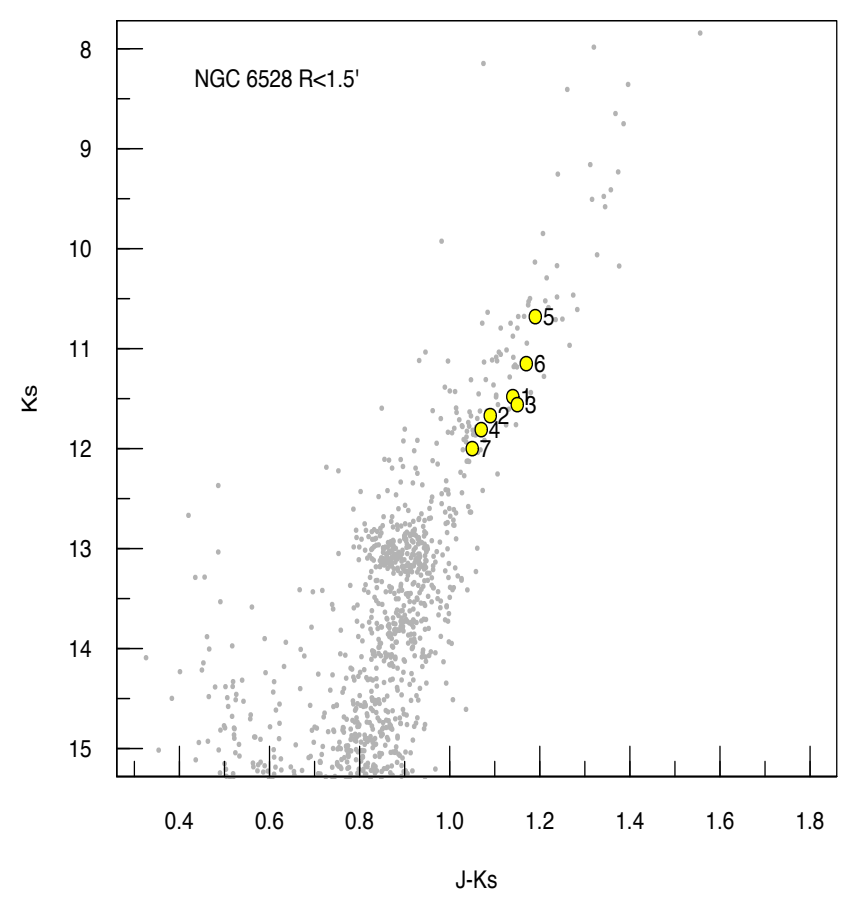

Fig. 2. CMD of NGC 6528 from the VVV survey corrected by the VVV reddening maps (Gonzalez et al. 2012). The yellow filled circles are our observed UVES sample.

We used equivalent widths (EWs) of the spectral lines to obtain the abundances for $\mathrm{Ca}, \mathrm{Ti}, \mathrm{Fe}, \mathrm{Co}$ and $\mathrm{Ni}$; the detailed explanation of the method we used to measure the EWs is given in Marino et al. (2008). Spectrum synthesis was used to determine the abundances of the other elements $(\mathrm{O}, \mathrm{Na}, \mathrm{Mg}, \mathrm{Al}, \mathrm{Si}$, $\mathrm{Sc}, \mathrm{V}, \mathrm{Cr}, \mathrm{Mn}, \mathrm{Cu}, \mathrm{Zr}, \mathrm{Ba}$ and $\mathrm{Eu}$ ), whose lines are affected by blending. We calculated five synthetic spectra of different abundance for each line, and estimated the best-fitting value as the one that minimizes the root mean square (rms) scatter. An example of this method is showed in Fig. 3 for two lines (oxygen and aluminum). Only lines not contaminated by telluric lines were used. The adopted solar abundances we used are reported in Table 3.

We performed an internal error analysis varying $T_{\text {eff }}, \log (\mathrm{g})$, $[\mathrm{Fe} / \mathrm{H}]$, and $v_{t}$ and redetermining abundances of each element for star \#3 of our sample, assumed to be representative of the entire sample. Parameters were varied by $\Delta T_{\text {eff }}=+41 \mathrm{~K}, \Delta \log (\mathrm{g})=$ $+0.14 \mathrm{dex}, \Delta[\mathrm{Fe} / \mathrm{H}]=+0.05 \mathrm{dex}$, and $\Delta v_{t}=+0.13 \mathrm{~km} \mathrm{~s}^{-1}$, which we estimated as our typical internal errors. The amount of variation of the parameter was calculated using three stars 

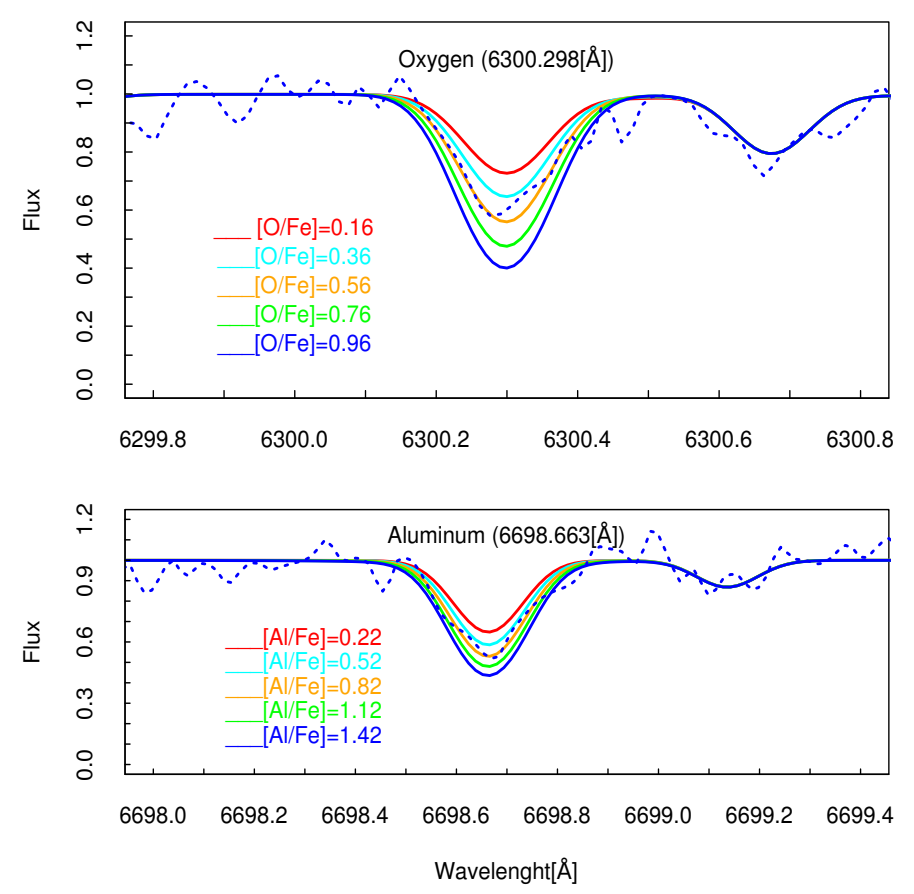

Fig. 3. Spectrum synthesis fits for oxygen (star \#5) and aluminum (star \#5) lines, respectively. The dotted line is the observed spectrum and the solid color lines show the synthesized spectra corresponding to different abundances.

representative of our sample (\#1, \#3, and \#4) with relatively low, intermediate, and high effective temperature, respectively, according to the procedure that was performed by Marino et al. (2008), which we follow in this study. Moreover, the procedure was used previously in several studies with excellent results (Muñoz et al. 2013, 2017; Mura-Guzmán et al. 2018).

The error introduced by the uncertainty on the EW $\left(\sigma_{S / N}\right)$ was calculated by dividing the rms scatter by the square root of the number of the lines used for a given element and a given star. For elements whose abundance was obtained by spectrum synthesis, the error is given in the output of the fitting procedure.

Finally, the error for each $[\mathrm{X} / \mathrm{Fe}]$ ratio as a result of uncertainties in atmospheric parameters and $\sigma_{S / N}$ is listed in Table 4. The total internal error $\left(\sigma_{\text {tot }}\right)$ is given by:

$\sigma_{\text {tot }}=\sqrt{\sigma_{T_{\text {eff }}^{2}}^{2}+\sigma_{\log (g)}^{2}+\sigma_{v_{t}}^{2}+\sigma_{[\mathrm{Fe} / \mathrm{H}]}^{2}+\sigma_{S / N}^{2}}$,

In Table 4 we compare the total internal error for each element with the observed error (standard deviation of the sample).

\section{Results}

Here we discuss our results in detail, compare them with previous studies of this cluster, and also make a general comparison with other bulge globular clusters analyzed up to now.

\subsection{Variability}

We have checked the variability of all our stars using the VVV survey data (Minniti et al. 2010; Saito et al. 2012), which gives us multicolor photometry in five bands: $Z$ (0.87 um), $Y$ (1.02 um), $J(1.25 \mathrm{um}), H(1.64 \mathrm{um})$ and $K_{s}(2.14 \mathrm{um})$. The survey covers an area of 562 square degrees, with 196 tiles that cover the bulge and 152 covering the adjacent southern plane.

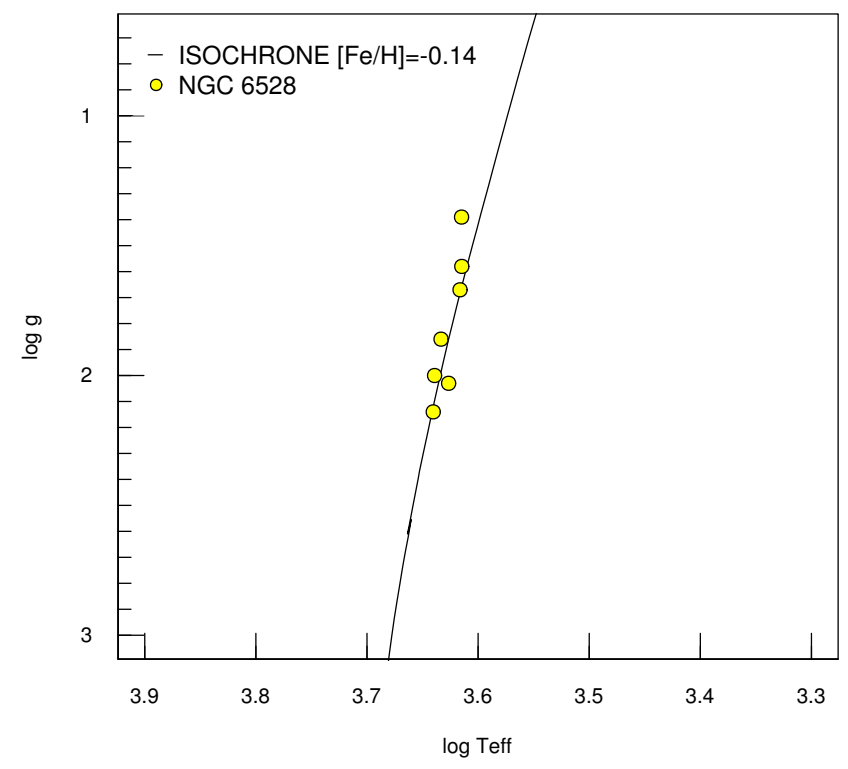

Fig. 4. $\log$ g vs. $\log T_{\text {eff }}$ for our sample of seven stars. The overplotted isochrone has a metallicity of $-0.14 \mathrm{dex},[\alpha / \mathrm{Fe}]=+0.20 \mathrm{dex}$ and age of $11 \mathrm{Gyr}$ (Dotter et al. 2008).

NGC 6528 is located on tile b278 whose central coordinates are RA: 18:04:40.94 Dec: -30:13:18.5 (J2000). The VVV catalogue of aperture photometry for each epoch was obtained from the Cambridge Astronomical Survey Unit $\left(\mathrm{CASU}^{2}\right)$. The catalog has positions, fluxes and flags which indicate the most probable morphological classification. In this last case, the flag "-1", which was chosen for this analysis, denotes the best-quality photometry of stellar objects, other flags in the catalog are " 0 " (noise), “-2" (borderline stellar), “-7” (sources containing bad pixels), and "-9" (saturated source).

In order to detect any periodic signals, we computed the generalized Lomb Scargle (GLS; Zechmeister \& Kürster 2009) and phase dispersion minimization (PDM; Stellingwerf 1978) algorithms of each star using aperture photometry. As a result, we detected significant variability in star \#5.

After this previous confirmation, we verified the variability in this star using point spread function (PSF) photometry. Because the time series contains multiple points for each epoch, we performed the average for epochs with a few seconds of separation. We obtained a total of 73 epochs in the $K_{s}$ band from April 2010 to September 2015, in a range of $10.6311<K_{s}<$ $10.7035 \mathrm{mag}$. We found a period of 0.25729 days, as shown in Fig. 5. The amplitude of the light curve was determined by the Fourier fit, yielding $0.05 \mathrm{mag}$.

We could not identify the type of variable, since it is difficult to classify variability type, due to the few features showed in $K_{s}$ bands (Catelan et al. 2013; Alonso-García et al. 2015).

\subsection{Iron}

We found a mean $[\mathrm{Fe} / \mathrm{H}]$ value for all our sample of seven stars of $[\mathrm{Fe} / \mathrm{H}]=-0.20 \pm 0.06$ dex. However, the scatter observed is $\sigma_{\text {obs }}=0.16$, which is significantly larger than the total expected spread of only 0.05 dex and is thus not due to errors in the atmospheric parameters, which are small in comparison with the scatter (see Table 4). However, it is important to note that our sample is small, only seven stars, and therefore does allow a statistically

\footnotetext{
2 http://apm49.ast.cam.ac.uk/
} 
Table 3. Abundances of the observed stars.

\begin{tabular}{|c|c|c|c|c|c|c|c|c|c|}
\hline Elements & 1 & 2 & 3 & 4 & 5 & 6 & 7 & Cluster & Sun \\
\hline \multirow[t]{2}{*}[\mathrm{O}/\mathrm{Fe}]{} & 0.07 & 0.00 & 0.31 & 0.15 & 0.11 & -0.09 & -0.09 & $+0.07 \pm 0.05$ & 8.83 \\
\hline & \pm 0.03 & \pm 0.04 & \pm 0.04 & \pm 0.06 & \pm 0.05 & \pm 0.03 & \pm 0.05 & & \\
\hline \multirow[t]{2}{*}[\mathrm{Na}/\mathrm{Fe}]{$_{\mathrm{NLTE}}$} & 0.10 & -0.02 & 0.31 & 0.75 & 0.82 & 0.60 & 0.19 & $+0.39 \pm 0.13$ & 6.32 \\
\hline & \pm 0.08 & \pm 0.07 & \pm 0.06 & \pm 0.07 & \pm 0.06 & \pm 0.04 & \pm 0.07 & & \\
\hline \multirow[t]{2}{*}[\mathrm{Mg}/\mathrm{Fe}]{} & 0.34 & 0.37 & 0.24 & 0.20 & 0.29 & 0.28 & 0.11 & $+0.26 \pm 0.03$ & 7.56 \\
\hline & \pm 0.03 & \pm 0.05 & \pm 0.05 & \pm 0.06 & \pm 0.07 & \pm 0.04 & \pm 0.06 & & \\
\hline \multirow[t]{2}{*}[\mathrm{Al}/\mathrm{Fe}]{} & 0.44 & 0.32 & 0.54 & 0.56 & 0.49 & 0.40 & 0.33 & $+0.44 \pm 0.04$ & 6.43 \\
\hline & \pm 0.04 & \pm 0.04 & \pm 0.04 & \pm 0.05 & \pm 0.07 & \pm 0.04 & \pm 0.05 & & \\
\hline \multirow[t]{2}{*}[\mathrm{Si}/\mathrm{Fe}]{} & 0.16 & -0.17 & -0.06 & 0.20 & -0.10 & -0.07 & -0.12 & $-0.02 \pm 0.05$ & 7.61 \\
\hline & \pm 0.08 & \pm 0.06 & \pm 0.06 & \pm 0.07 & \pm 0.06 & \pm 0.04 & \pm 0.08 & & \\
\hline \multirow[t]{2}{*}[\mathrm{Ca}/\mathrm{Fe}]{} & 0.01 & -0.10 & 0.02 & 0.02 & 0.10 & 0.21 & -0.05 & $+0.03 \pm 0.04$ & 6.39 \\
\hline & \pm 0.07 & \pm 0.06 & \pm 0.04 & \pm 0.06 & \pm 0.05 & \pm 0.05 & \pm 0.06 & & \\
\hline \multirow[t]{2}{*}[\mathrm{Sc}/\mathrm{Fe}]{} & 0.32 & 0.22 & 0.38 & 0.41 & -0.12 & 0.04 & 0.11 & $+0.19 \pm 0.07$ & 3.12 \\
\hline & \pm 0.07 & \pm 0.06 & \pm 0.06 & \pm 0.07 & \pm 0.07 & \pm 0.04 & \pm 0.07 & & \\
\hline \multirow[t]{2}{*}[\mathrm{Ti}/\mathrm{Fe}]{} & 0.44 & 0.24 & 0.47 & 0.52 & 0.60 & 0.73 & 0.45 & $+0.49 \pm 0.06$ & 4.94 \\
\hline & \pm 0.03 & \pm 0.04 & \pm 0.04 & \pm 0.06 & \pm 0.05 & \pm 0.06 & \pm 0.06 & & \\
\hline \multirow[t]{2}{*}[\mathrm{V}/\mathrm{Fe}]{} & 0.13 & 0.17 & 0.22 & 0.19 & 0.33 & 0.25 & 0.23 & $+0.22 \pm 0.02$ & 4.00 \\
\hline & \pm 0.06 & \pm 0.06 & \pm 0.06 & \pm 0.06 & \pm 0.05 & \pm 0.04 & \pm 0.06 & & \\
\hline \multirow[t]{2}{*}[\mathrm{Cr}/\mathrm{Fe}]{} & 0.07 & -0.07 & 0.31 & 0.10 & 0.18 & 0.26 & -0.03 & $+0.12 \pm 0.05$ & 5.63 \\
\hline & \pm 0.10 & \pm 0.10 & \pm 0.10 & \pm 0.11 & \pm 0.06 & \pm 0.06 & \pm 0.08 & & \\
\hline \multirow[t]{2}{*}[\mathrm{Mn}/\mathrm{Fe}]{} & 0.40 & 0.04 & 0.27 & 0.04 & 0.45 & 0.17 & 0.08 & $+0.21 \pm 0.06$ & 5.37 \\
\hline & \pm 0.09 & \pm 0.07 & \pm 0.09 & \pm 0.09 & \pm 0.07 & \pm 0.08 & \pm 0.09 & & \\
\hline \multirow[t]{2}{*}[\mathrm{Fe}/\mathrm{H}]{} & -0.16 & -0.12 & -0.24 & -0.11 & -0.55 & -0.20 & -0.03 & $-0.20 \pm 0.06$ & 7.50 \\
\hline & \pm 0.03 & \pm 0.02 & \pm 0.02 & \pm 0.03 & \pm 0.04 & \pm 0.03 & \pm 0.03 & & \\
\hline \multirow[t]{2}{*}[\mathrm{Co}/\mathrm{Fe}]{} & 0.74 & 0.52 & 0.64 & 0.61 & 0.54 & 0.83 & 0.53 & $+0.63 \pm 0.04$ & 4.93 \\
\hline & \pm 0.08 & \pm 0.08 & \pm 0.09 & \pm 0.10 & \pm 0.09 & \pm 0.08 & \pm 0.10 & & \\
\hline \multirow[t]{2}{*}[\mathrm{Ni}/\mathrm{Fe}]{} & 0.25 & 0.15 & 0.22 & 0.16 & 0.13 & 0.41 & 0.22 & $+0.22 \pm 0.04$ & 6.26 \\
\hline & \pm 0.05 & \pm 0.06 & \pm 0.08 & \pm 0.06 & \pm 0.06 & \pm 0.08 & \pm 0.08 & & \\
\hline \multirow[t]{2}{*}[\mathrm{Cu}/\mathrm{Fe}]{} & 0.15 & 0.01 & 0.49 & 0.31 & 0.44 & 0.47 & 0.52 & $+0.34 \pm 0.07$ & 4.19 \\
\hline & \pm 0.09 & \pm 0.11 & \pm 0.12 & \pm 0.13 & \pm 0.10 & \pm 0.10 & \pm 0.12 & & \\
\hline \multirow[t]{2}{*}[\mathrm{Zr}/\mathrm{Fe}]{} & -0.19 & -0.22 & -0.14 & -0.17 & -0.05 & -0.19 & -0.15 & $-0.16 \pm 0.02$ & 2.25 \\
\hline & \pm 0.05 & \pm 0.04 & \pm 0.06 & \pm 0.06 & \pm 0.04 & \pm 0.04 & \pm 0.06 & & \\
\hline \multirow[t]{2}{*}[\mathrm{Ba}/\mathrm{Fe}]{} & 0.04 & 0.07 & 0.08 & 0.06 & 0.00 & 0.08 & 0.10 & $+0.06 \pm 0.01$ & 2.34 \\
\hline & \pm 0.06 & \pm 0.05 & \pm 0.06 & \pm 0.07 & \pm 0.04 & \pm 0.04 & \pm 0.06 & & \\
\hline \multirow[t]{2}{*}[\mathrm{Eu}/\mathrm{Fe}]{} & 0.30 & 0.25 & 0.37 & 0.31 & 0.13 & 0.16 & 0.26 & $+0.25 \pm 0.03$ & 0.52 \\
\hline & \pm 0.04 & \pm 0.05 & \pm 0.05 & \pm 0.05 & \pm 0.04 & \pm 0.04 & \pm 0.06 & & \\
\hline
\end{tabular}

Notes. Columns 2-8: abundances of the observed stars in NGC 6528. Column 9: mean abundance for the cluster; we include the statistical errors obtained from the mean. Column 10: abundances adopted for the Sun in this paper, indicated as $\log \epsilon($ El.). The errors presented for each abundance, in Cols. 2-8, were calculated by dividing the rms scatter by the square root of the number of the lines used for a given element and a given star. For elements whose abundance was obtained by spectrum synthesis, the error is the output of the fitting procedure.

robust analysis. Moreover, one of the stars shows a very extreme metallicity compared to the others (star \#5; see Table 1). This star is indeed the variable candidate (see Fig. 5 and Sect. 4.1). It is quite possible that the variability has affected the measurement of the iron abundance of this star in some way, causing a large offset with respect to the cluster mean. Another detailed spectroscopic analysis of this star is needed to investigate possible variability effects on the metallicity derivation. We note that, according to its radial velocity, position in the CMD, and atmospheric parameters, this star is very likely a cluster member.

Nonetheless, if we remove star \#5 from the sample, assuming that it is an outlier (presumably due to the variability), we obtain a metallicity average of $[\mathrm{Fe} / \mathrm{H}]=-0.14 \pm 0.03$ dex and a scatter of 0.07 , which is generally what is expected from errors alone. Therefore, we need a larger sample to conclusively prove or disprove an intrinsic metallicity spread. We note that Mauro et al. (2014) found a mean of $[\mathrm{Fe} / \mathrm{H}]=-0.18 \pm 0.14$ dex with a spread of 0.20 dex using low-resolution CaT spectra together with NIR photometry for a similar sample of stars (see Table 2), and suggested a possible intrinsic variation; however, their uncertainties are larger than ours. We note that they found star \#5 to have a metallicity that is almost identical to their mean value.

Comparing our results with Saviane et al. (2012) we note that we have three stars in common (see Table 2). Star \#7 shows a significant difference between the two studies while the other two stars do not. If we use the metallicity scale of Dias et al. (2016a,b) for NGC 6528 the average is $[\mathrm{Fe} / \mathrm{H}]=-0.13 \pm 0.07$, in excellent agreement with our results.

Schiavon et al. (2017) found a metallicity of -0.19 dex in two stars. Origlia et al. (2005) measured the abundance of four stars using near-infrared spectroscopy and found a mean metallicity of -0.17 dex. Zoccali et al. (2004) derived a value of -0.10 dex in three stars and Harris (1996) quotes a metallicity of -0.11 dex for NGC 6528. Liu et al. (2017) found a metallicity of 0.04 dex with a scatter of 0.07 dex, and their $\mathrm{S} / \mathrm{N}$ is similar to ours. Moreover, we have three stars in common (see Table 2), 
Table 4. Estimated errors on abundances for the representative star of our sample (star \#3); due to errors on atmospherics parameters and to spectral noise, compared with the observed errors.

\begin{tabular}{lccccccc}
\hline \hline $\mathrm{ID}$ & $\Delta T_{\text {eff }}=41 \mathrm{~K}$ & $\Delta \log (\mathrm{g})=0.14$ & $\Delta v_{t}=0.13$ & $\Delta[\mathrm{Fe} / \mathrm{H}]=0.05$ & $\sigma_{S / N}$ & $\sigma_{\text {tot }}$ & $\sigma_{\text {obs }}$ \\
\hline$\Delta([\mathrm{O} / \mathrm{Fe}])$ & -0.02 & 0.03 & -0.06 & -0.04 & 0.04 & 0.09 & 0.14 \\
$\Delta([\mathrm{Na} / \mathrm{Fe}])$ & 0.04 & -0.03 & -0.04 & -0.04 & 0.06 & 0.10 & 0.33 \\
$\Delta([\mathrm{Mg} / \mathrm{Fe}])$ & 0.05 & 0.05 & 0.07 & 0.05 & 0.05 & 0.12 & 0.09 \\
$\Delta([\mathrm{Al} / \mathrm{Fe}])$ & 0.03 & 0.02 & 0.05 & -0.02 & 0.04 & 0.08 & 0.10 \\
$\Delta([\mathrm{Si} / \mathrm{Fe}])$ & 0.05 & 0.06 & 0.04 & 0.04 & 0.06 & 0.11 & 0.14 \\
$\Delta([\mathrm{Ca} / \mathrm{Fe}])$ & -0.01 & 0.01 & 0.05 & -0.01 & 0.04 & 0.07 & 0.10 \\
$\Delta([\mathrm{Sc} / \mathrm{Fe}])$ & 0.03 & -0.05 & 0.00 & 0.04 & 0.06 & 0.09 & 0.19 \\
$\Delta([\mathrm{Ti} / \mathrm{Fe}])$ & -0.01 & -0.01 & 0.05 & -0.01 & 0.04 & 0.07 & 0.15 \\
$\Delta([\mathrm{V} / \mathrm{Fe}])$ & 0.02 & 0.07 & 0.05 & 0.06 & 0.06 & 0.12 & 0.06 \\
$\Delta([\mathrm{Cr} / \mathrm{Fe}])$ & 0.00 & 0.00 & 0.05 & 0.00 & 0.10 & 0.11 & 0.14 \\
$\Delta([\mathrm{Mn} / \mathrm{Fe}])$ & 0.00 & 0.03 & 0.06 & -0.05 & 0.09 & 0.12 & 0.17 \\
$\Delta([\mathrm{Fe} / \mathrm{H}])$ & 0.01 & 0.02 & 0.03 & 0.03 & 0.02 & 0.05 & 0.16 \\
$\Delta([\mathrm{Co} / \mathrm{Fe}])$ & 0.02 & -0.03 & 0.06 & 0.02 & 0.09 & 0.12 & 0.12 \\
$\Delta([\mathrm{Ni} / \mathrm{Fe}])$ & 0.02 & -0.03 & 0.05 & 0.02 & 0.08 & 0.10 & 0.09 \\
$\Delta([\mathrm{Cu} / \mathrm{Fe}])$ & 0.12 & 0.06 & 0.05 & 0.10 & 0.12 & 0.21 & 0.19 \\
$\Delta([\mathrm{Zr} / \mathrm{Fe}])$ & 0.11 & 0.07 & 0.06 & 0.08 & 0.17 & 0.12 & 0.05 \\
$\Delta([\mathrm{Ba} / \mathrm{Fe}])$ & 0.05 & 0.04 & 0.06 & -0.01 & 0.06 & 0.11 & 0.03 \\
$\Delta([\mathrm{Eu} / \mathrm{Fe}])$ & 0.01 & 0.06 & 0.03 & 0.03 & 0.05 & 0.09 & 0.08 \\
\hline
\end{tabular}
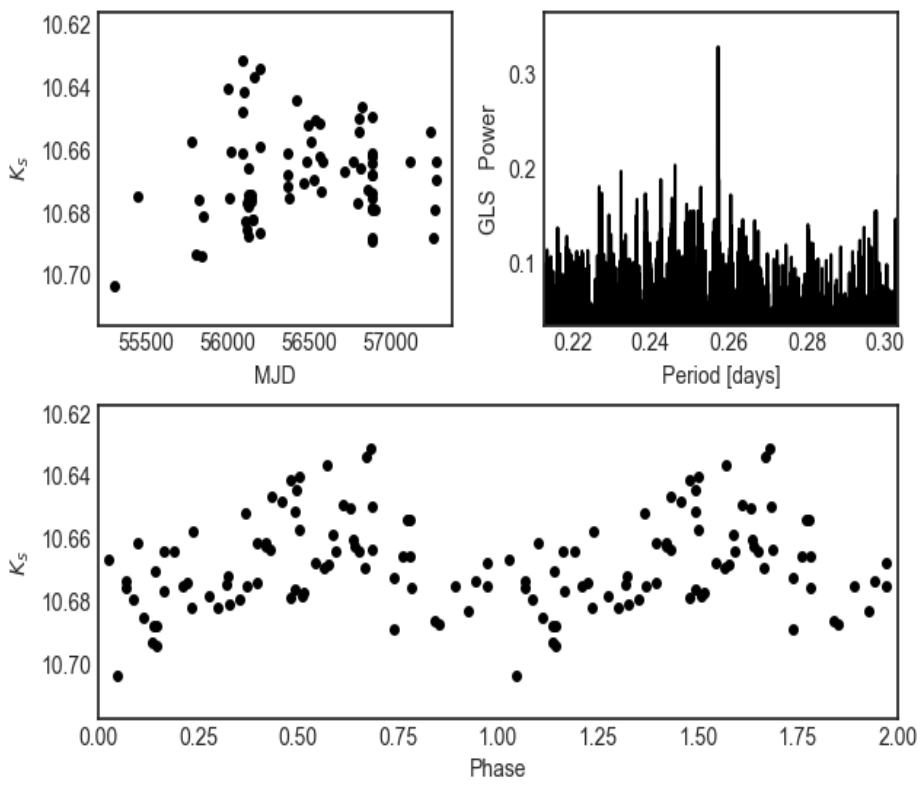

Fig. 5. Top left panel: light curve of the candidate variable star. Top right panel: generalized Lomb Scargle periodogram shows a strong peak at 0.25729 days. Bottom panel: phased light curve of the candidate variable star.

with good agreement for stars \#3 and \#6. Again, their value for star \#5 is not unusual compared to their other two stars. Furthermore, Carretta et al. (2001) measured the metallicity in four RHB stars in NGC 6528, and found a metallicity of 0.07 dex with total errors of $\sim 0.1$ dex. In general, we conclude that our mean metallicity is in good agreement with previous results. Even if $[\mathrm{Fe} / \mathrm{H}]$ for star \#5 is lower than the mean, the [El./Fe] ratio for this star agrees with the rest of the sample.

\subsection{Iron-peak elements}

The iron peak elements, although they have not been widely studied in bulge GCs, are very useful tools to better understand their chemical evolution. They are produced mainly by supernovae ( $\mathrm{SNe}$ ) and massive stars, the most important polluters of the interstellar medium (ISM).

In this study, we have measured the abundance of six ironpeak elements: Fe, Sc, V, Cr, Mn and Ni (see Table 3 and Fig. 6). Here we analyze the other elements besides Fe.

In Fig. 6 we plot iron-peak element abundance versus [Fe/H], comparing with two bulge GCs, NGC 6553 (Tang et al. 2017) and NGC 6440 (Muñoz et al. 2017), from our previous study. We find for NGC 6528 a super-solar abundance for all the iron peak elements, although slightly more moderate for the case of $\mathrm{V}$ and Ni. In general, NGC 6528 shows good agreement with NGC 6553 (Tang et al. 2017) and with bulge field stars from Barbuy et al. (2013) and Johnson et al. (2014).

Although we do not have bulge field star measurements for Sc or V, we notice that the stars of NGC 6528 follow the Galactic disk field-star trend but at higher abundance, which is typical behavior of bulge stars.

We found an overabundance in $[\mathrm{Mn} / \mathrm{Fe}]=0.21 \pm 0.06 \mathrm{dex}$ for NGC 6528 (see Fig. 6). This overabundance of Mn is an indication of a formation site rich in $\mathrm{Mn}$, which is mainly produced in SNeIa (Iwamoto et al. 1999; Kobayashi et al. 2006; Cescutti et al. 2008). Nickel shows in general very good agreement with the bulge field and with NGC 6553.

The nucleosynthetic origin of copper is not very clear (McWilliam \& Smecker-Hane 2005). Some authors suggest SNeIa do not contribute significantly to $\mathrm{Cu}$ synthesis (Shetrone et al. 2003). Nevertheless, Matteucci et al. (1993) and Mishenina et al. (2002) indicate that SNeIa could be an important producer of copper. In the case of NGC 6528, we found very good agreement with NGC 6553 and bulge field stars (See Fig. 6).

In summary, NGC 6528 shows good agreement with NGC 6553 for each element compared $(\mathrm{Cr}, \mathrm{Ni}$ and $\mathrm{Cu})$ and good agreement with bulge field stars as well. The super-solar value found for all iron-peak elements measured in this study could be an indication of a strong early pollution by SN explosions, mainly those of SNeIa. However, the detailed origins of many of these elements remain unclear. 


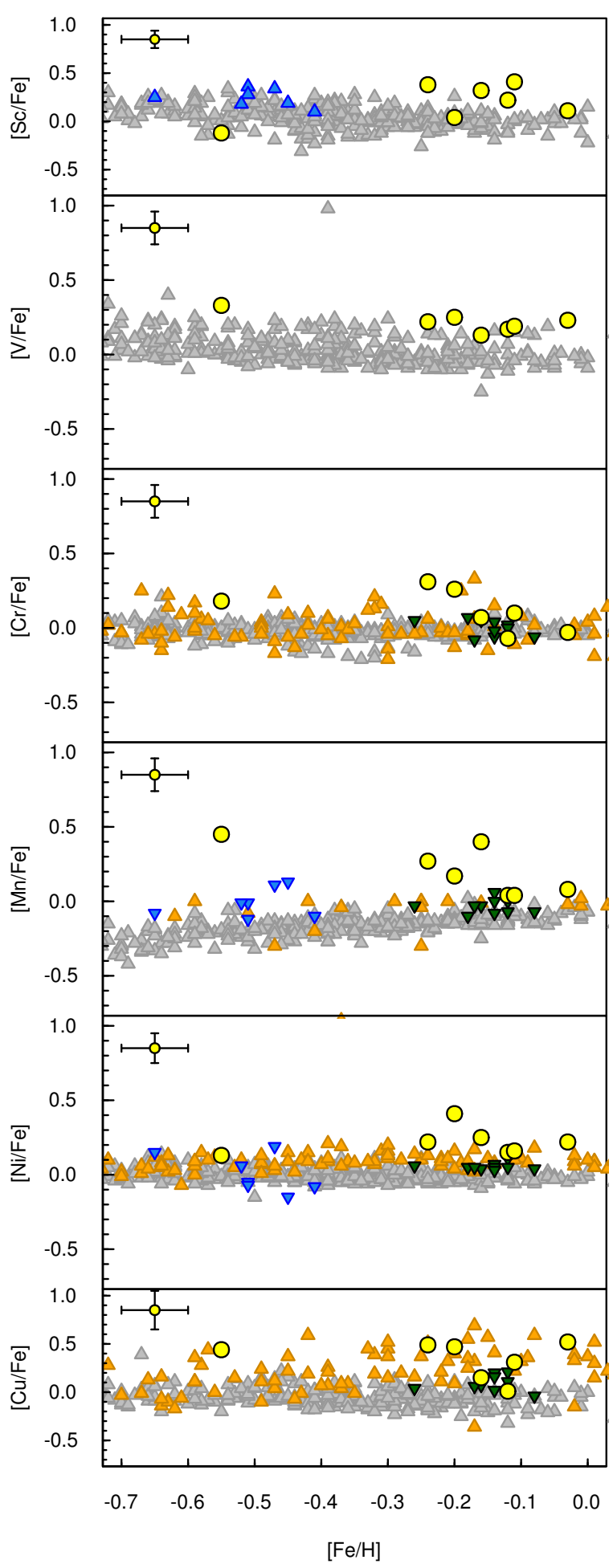

Fig. 6. $[\mathrm{Sc}, \mathrm{V}, \mathrm{Cr}, \mathrm{Mn}, \mathrm{Ni}, \mathrm{Cu} / \mathrm{Fe}]$ vs. [Fe/H]. Filled yellows circles are our data for NGC 6528, filled blue triangles: NGC 6440 (Muñoz et al. 2017), filled orange triangles: Bulge field stars (Barbuy et al. 2013; Johnson et al. 2014), filled gray triangles: halo and disk stars (Fulbright 2000; François et al. 2007; Reddy et al. 2003, 2006), filled dark green triangles: NGC 6553 (Johnson et al. 2014).

Carretta et al. (2001) derived the abundances for the ironpeak elements ( $\mathrm{Sc}, \mathrm{V}, \mathrm{Cr}, \mathrm{Mn}$, and $\mathrm{Ni}$ ) in four RHB stars. Comparing the results of this latter study with ours, we find a significative difference. Taking into account the errors in both studies, however, we find compatibility between $\mathrm{Sc}, \mathrm{Cr}$, and $\mathrm{Ni}$ (see Fig. 13).
Moreover, although our findings show good compatibility with the results of Barbuy et al. (2016), they concluded that abundances of HP1 agree with enrichment by SNII at an early epoch, whereas our results show a good agreement with enrichment by SNIa, which happens later. These results are an indication that NGC6528 is younger that HP1, which would be expected in light of its metallicity. In fact, the age measurement by Ortolani et al. (2011) for HP1 is $\sim 13 \mathrm{Gyr}$ and Lagioia et al. (2014) found an age of $\sim 11$ Gyr for NGC 6528.

\section{4. $\alpha$ elements}

We measured five $\alpha$ elements $(\mathrm{O}, \mathrm{Mg}, \mathrm{Si}, \mathrm{Ca}$ and $\mathrm{Ti})$ and found that the abundances for $\mathrm{O}, \mathrm{Si}$, and $\mathrm{Ca}$ listed in Table 3 (see Fig. 7) are essentially solar. However, $\mathrm{Mg}$ and $\mathrm{Ti}$ are definitively enhanced (see Table 3 and Fig. 7). Using $\mathrm{Mg}, \mathrm{Si}, \mathrm{Ca}$, and $\mathrm{Ti}$ to estimate a mean $\alpha$ abundance, we obtain $[\alpha / \mathrm{Fe}]=0.19 \pm 0.03$. Origlia et al. (2005) using NIR spectroscopy for four members of NGC 6528 found the value $[\alpha / \mathrm{Fe}] \simeq+0.33 \pm 0.01$, higher than our value. They obtain the $\alpha$ abundances using the same elements used in this study ( $\mathrm{Mg}, \mathrm{Si}, \mathrm{Ca}$ and $\mathrm{Ti})$. Carretta et al. (2001) measured the $\alpha$ abundances in their sample of RHB stars, and their values are higher than ours, in particular in the case of Titanium; the other elements $(\mathrm{Mg}, \mathrm{Si}$, and $\mathrm{Ca}$ ) are compatible considering the errors. Also, Zoccali et al. (2004) found a value $[\alpha / \mathrm{Fe}] \simeq+0.1$, consistent with our results (see Fig. 13).

It is interesting to note that NGC 6528 shows good agreement with the bulge field stars with regard to $\alpha$ elements. In the case of $\mathrm{O}, \mathrm{Si}$, and $\mathrm{Ca}$, the cluster follows the trend for the lower part of the field star distribution, while $\mathrm{Mg}$ is in the middle of this distribution and $\mathrm{Ti}$ is in the upper envelope; this is certainly indicative of a similar origin (see Sect. 5).

Notably, the typical $\alpha$ elements that generally show a substantial spread among GCs, $\mathrm{O}$, and $\mathrm{Mg}$ do not show evidence for an intrinsic variation in NGC 6528, very reminiscent of the behavior we found for the bulge GC NGC 6553 .

In general, we see that all the metal-rich bulge GCs that we include in this study (NGC 6440, NGC 6441, Terzan 5, NGC 6553 and NGC 6528) show good agreement with the tendency of the bulge field stars (See Figs. 7 and 8).

As mentioned in our work on NGC 6440 (Muñoz et al. 2017), the $\alpha$ elements in NGC 6528 also show a peculiar behavior. In the graph of $[\alpha / \mathrm{Fe}]$ versus $[\mathrm{Fe} / \mathrm{H}], \mathrm{NGC} 6528$ is located at a higher metallicity than the so-called knee, which marks the change between a high rate of pollution of the $\alpha$-elements from SNeII to a pollution coming mainly from SNeIa with less production of $\alpha$-elements. The patterns, which are observed in $\mathrm{O}$, $\mathrm{Si}$, and $\mathrm{Ca}$ would indicate that NGC 6528 was formed in an area mainly polluted by SNeIa. However, $\mathrm{Mg}$ and Ti remain at SNeII-enhanced levels despite the high metallicity of this GC. This argument is supported by Zoccali et al. (2004) for O, $\mathrm{Ca}$, and $\mathrm{Mg}$, by Carretta et al. (2001) for $\mathrm{O}$ and $\mathrm{Mg}$, and by Schiavon et al. (2017) for the case of Mg. On the other hand, the argument is not well supported by Origlia et al. (2005; see Fig. 13). It is worth noting nonetheless that for all the studies, including ours, the samples are too small for reliable conclusions to be made.

\subsection{Na-O anticorrelation}

The Na-O anticorrelation is the most famous among GCs, and is the prime evidence associated with MPs in GCs (Carretta et al. 2009b,a; Gratton et al. 2012). However, we note that at least one GC, Ruprecht 106, apparently does not show this anticorrelation, 


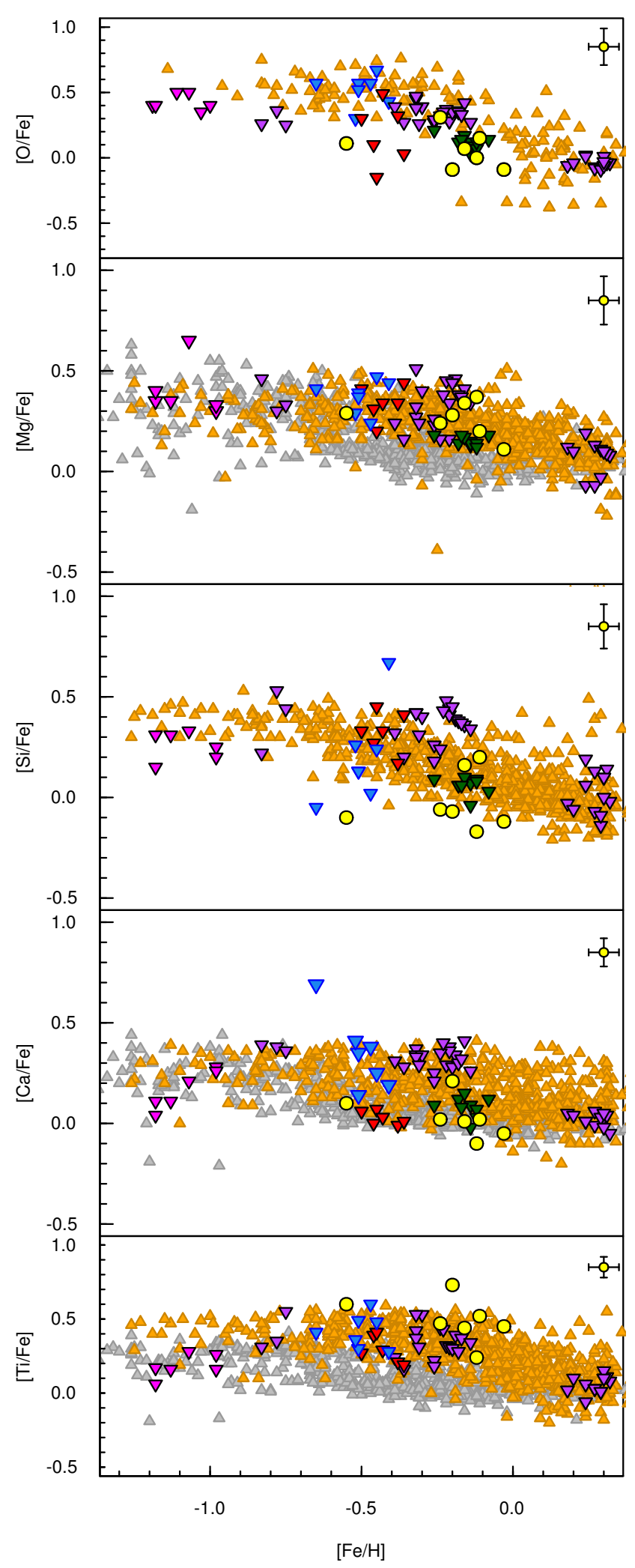

Fig. 7. $[\mathrm{O} / \mathrm{Fe}],[\mathrm{Mg} / \mathrm{Fe}],[\mathrm{Si} / \mathrm{Fe}],[\mathrm{Ca} / \mathrm{Fe}], \quad[\mathrm{Ti} / \mathrm{Fe}]$ vs. $[\mathrm{Fe} / \mathrm{H}]$ Filled yellow circles indicate our data for NGC 6528, filled blue triangles: NGC 6440 (Muñoz et al. 2017), filled red triangles: NGC 6441 (Gratton et al. 2006, 2007), filled purple triangles: Terzan 5 (Origlia et al. 2011, 2013), filled dark green triangles: NGC 6553 (Tang et al. 2017), filled magenta triangles: HP1 (Barbuy et al. 2016), filled orange triangles: bulge field stars (Gonzalez et al. 2012), filled gray triangles: halo and disk fields stars (Venn et al. 2004).

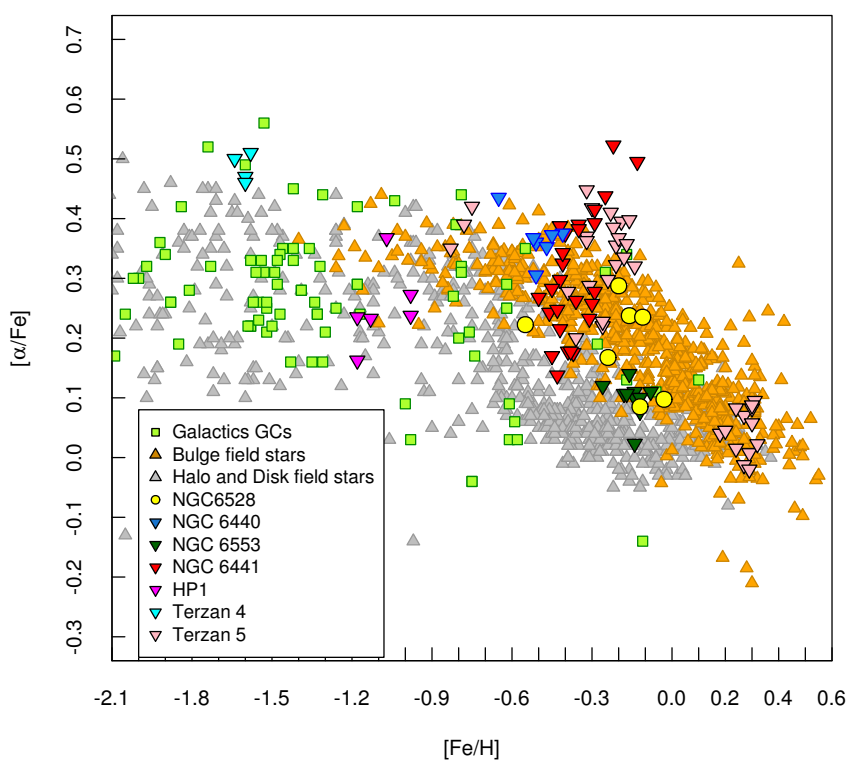

Fig. 8. $[\alpha / \mathrm{Fe}]$ vs. $[\mathrm{Fe} / \mathrm{H}]$. Filled yellow circles are our data for NGC 6528, filled blue triangles: NGC 6440 (Muñoz et al. 2017), filled red triangles: NGC 6441 (Gratton et al. 2006, 2007), filled pink triangles: Terzan 5 (Origlia et al. 2011, 2013), filled cyan triangles: Terzan 4 (Origlia \& Rich 2004), filled dark green triangles: NGC 6553 (Tang et al. 2017), filled magenta triangles: HP1 (Barbuy et al. 2016), filled orange triangles: bulge field stars (Gonzalez et al. 2012), filled green square: GCs from Pritzl et al. (2005), filled gray triangles: Halo and Disk fields stars (Venn et al. 2004).

although it is likely an extragalactic GC (Villanova et al. 2013). According to the models (Ventura \& D'Antona 2006), the GCs that show this anticorrelation form a first generation of stars followed by a second generation which is much more Na-rich and O-poor. The nature of the polluters and the details of the pollution process are currently hotly debated. We did not find an extended O-Na anticorrelation, especially in the case of oxygen. Comparing the scatter in our measurement, we find a significant intrinsic spread in $\mathrm{Na}\left(\sigma_{\mathrm{obs}}=0.33\right)$ while oxygen $\left(\sigma_{\mathrm{obs}}=0.14\right)$ is very similar to the expected observational errors $\left(\sigma_{\text {tot }}=0.09\right)$. This behavior is strange because commonly the Galactic GCs show a large spread in both oxygen and sodium.

Origlia et al. (2005) obtained the abundance of oxygen for four giants, and the average is $[\mathrm{O} / \mathrm{Fe}]=0.31$ dex without significant dispersion. Despite the fact that their value is higher than ours, both results are consistent taking into account the errors of both (see Fig. 13). Carretta et al. (2001) found a mean for oxygen of $[\mathrm{O} / \mathrm{Fe}]=0.07 \mathrm{dex}$ in three stars, and in the case of $\mathrm{Na}$, Carretta found a mean of $[\mathrm{Na} / \mathrm{Fe}]=0.40$ dex in four stars; their values are in excellent agreement with our findings (see Fig. 13). Zoccali et al. (2004) measured the abundance of oxygen for three RGB stars in NGC 6528. They found an average of $[\mathrm{O} / \mathrm{Fe}]=0.15$ dex with very low dispersion. In addition, they found a mean for $[\mathrm{Na} / \mathrm{Fe}]=0.43$ dex with a large dispersion. Although these results are for only three stars, their behavior is consistent with our results (see Fig. 13). Finally, Schiavon et al. (2017) report the chemical abundances for $\mathrm{Na}$ in two stars with a value of 0.23 dex and 0.61 dex. Despite the fact that only two stars are reported, these are in concordance with our measurement for Na (see Fig. 13).

When we study the $\mathrm{Na}-\mathrm{O}$ anti-correlation for additional bulge GCs (see Fig.9), we note that NGC 6528 (this study), 


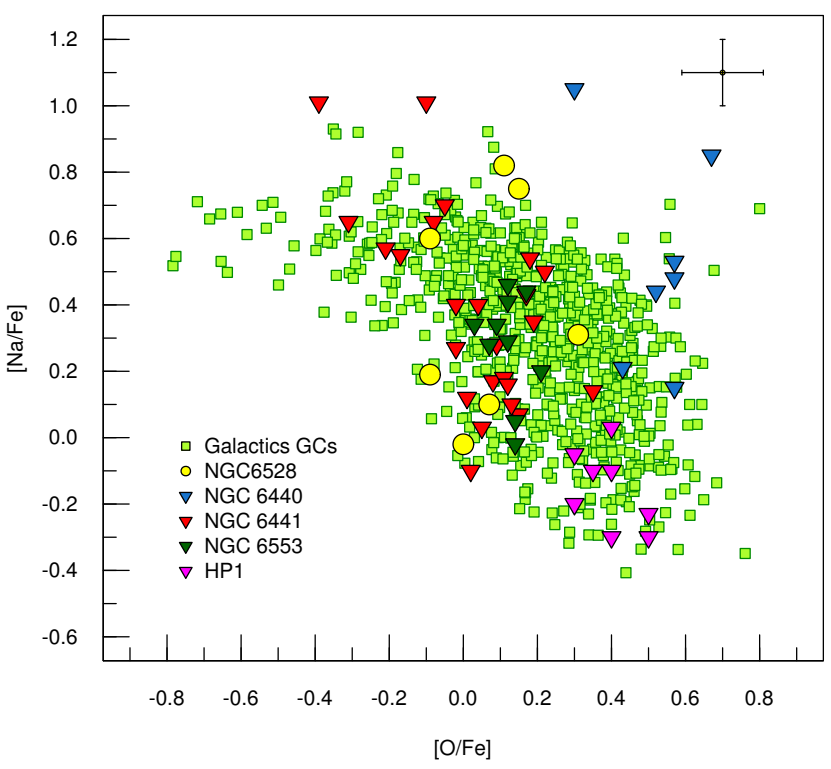

Fig. 9. $[\mathrm{O} / \mathrm{Fe}]$ vs. $[\mathrm{Na} / \mathrm{Fe}]$. Filled yellow circles are our data for NGC 6528, filled blue triangles: NGC 6440 (Muñoz et al. 2017), filled red triangles: NGC6441 (Gratton et al. 2006, 2007), filled dark green triangles: NGC 6553 (Tang et al. 2017), filled magenta triangles: HP1 (Barbuy et al. 2016), filled green square: Galactic GCs from Carretta et al. (2009a).

NGC 6440, NGC 6553, and HP1 show a vertical O-Na trend, marked by a significant spread in sodium, but not in oxygen. In fact, the bulge GCs show a low intrinsic oxygen scatter, consistent with no true spread. We must bear in mind that the samples are generally small. Therefore, we need to check this behavior with a larger sample. If this trend holds, it would highlight a chemical evolution difference in the bulge GCs compared to those of the typical halo.

\section{6. $M g-A /$}

Carretta et al. (2009a) found a Mg-Al anticorrelation in a large sample of Galactic GCs. The Mg-Al chain plays an important role in this correlation, however, very high temperatures are necessary to achieve it. Aluminum shows a large spread in many Galactic GCs, particularly those with a metallicity lower than $[\mathrm{Fe} / \mathrm{H}]=-1.1$ dex (Mészáros et al. 2015). Also, Pancino et al. (2017) verify that the $\mathrm{Mg}$ - $\mathrm{Al}$ anticorrelation extension depends on cluster mass and metallicity.

Carretta et al. (2001) measured the abundances of magnesium in three RHB stars. The average obtained was $[\mathrm{Mg} / \mathrm{Fe}]=$ 0.14 dex. Origlia et al. (2005) obtained the abundances of magnesium in four giants stars, obtaining an average of $[\mathrm{Mg} / \mathrm{Fe}]=$ 0.35 dex. Zoccali et al. (2004) found a mean of $[\mathrm{Mg} / \mathrm{Fe}]=$ 0.07 dex for magnesium in three stars. Finally, Schiavon et al. (2017) reported the chemical abundances for magnesium and aluminum in two stars, finding averages of $[\mathrm{Mg} / \mathrm{Fe}]=0.15 \mathrm{dex}$ and $[\mathrm{Al} / \mathrm{Fe}]=0.28$ dex. These studies have small samples or in other cases have measurements only for magnesium. In this context our results are compatible with these studies (see Fig. 13).

We find no Mg-Al anticorrelation in NGC 6528 (see Fig. 10). Indeed, we find no significant spread in $\mathrm{Al}\left(\sigma_{\mathrm{obs}}=0.10\right)$ or in $\mathrm{Mg}\left(\sigma_{\mathrm{obs}}=0.09\right)$, similar to the cases of NGC 6553 and NGC 5927 (Mura-Guzmán et al. 2018), other metal-rich GCs from the disk, but in contrast with NGC 6440, which does show a Mg-Al anticorrelation. From our study (Muñoz et al. 2017),

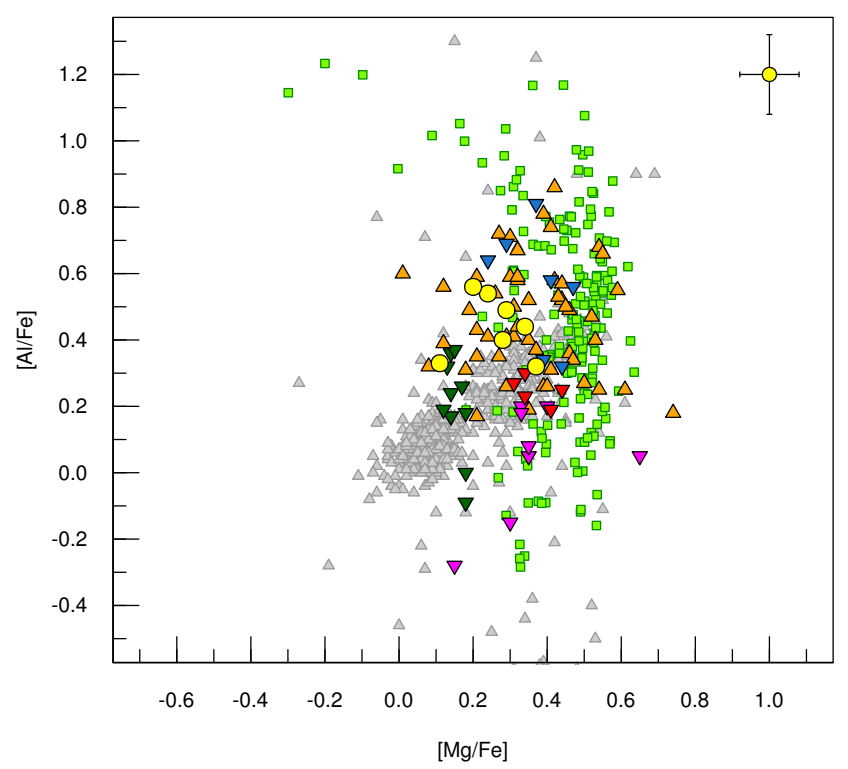

Fig. 10. $[\mathrm{Mg} / \mathrm{Fe}]$ vs. $[\mathrm{Al} / \mathrm{Fe}]$. Filled yellow circles are our data for NGC 6528, filled blue triangles: NGC 6440 (Muñoz et al. 2017), filled red triangles: NGC6441 (Gratton et al. 2006), filled yellow triangles: Terzan 5 (Origlia et al. 2011), filled dark green triangles: NGC 6553 (Tang et al. 2017), filled magenta triangles: HP1 (Barbuy et al. 2016), filled orange triangles: bulge field stars (Lecureur et al. 2007), filled green square: GCs from Carretta et al. (2009a), filled gray triangles: halo and disk fields stars (Fulbright 2000; Reddy et al. 2003, 2006; Barklem et al. 2005; Cayrel et al. 2004).

this bulge globular cluster does not show a large extension in the $\mathrm{Mg}-\mathrm{Al}$ anticorrelation, but it has a significant spread in $\mathrm{Al}$ $\left(\sigma_{\text {obs }}=0.18\right)$, unlike NGC 6528 .

\subsection{Neutron-capture elements}

Analyzing neutron-capture elements helps us to better understand the processes involved in the creation of MPs in GCs. For example, low-mass AGB stars produce mainly s-elements like Ba (Gallino et al. 1998; Straniero et al. 2006), and SNeII explosions are associated mainly with $r$-process element production.

We measured three heavy elements: $\mathrm{Zr}$, Ba and $\mathrm{Eu}$. We find that the abundance of Ba for NGC 6528 is slightly supersolar $([\mathrm{Ba} / \mathrm{Fe}]=0.06 \mathrm{dex})$, showing a similar value to that of NGC 6441 (see Fig. 11). Carretta et al. (2001) obtained the Ba abundances in four RHB stars in good agreement with our results (see Fig. 13).

For $[\mathrm{Eu} / \mathrm{Fe}]$, the bulge field stars show a decrease with $[\mathrm{Fe} / \mathrm{H}]$. The decrease is due to the production of iron by SNeIa (Ballero et al. 2007). In the case of europium, we find a supersolar value of $([\mathrm{Eu} / \mathrm{Fe}]=0.25)$, in very good agreement with the bulge field-star trend and with other bulge GCs including NGC 6440 and NGC 6441 (see Fig. 11).

In Fig. 12 we observe $[\mathrm{Ba} / \mathrm{Eu}]$ versus $[\mathrm{Fe} / \mathrm{H}]$, which allows us to establish the relative importance of the $s$-process versus the $r$-process. In the case of NGC 6528, we find a value of [Ba/Eu] subsolar, similar to the values shown by NGC 6441, which suggests that the heavy elements were produced mainly by explosive events in SNeII. We also notice very good agreement with the bulge (see Fig. 12).

Our results are compatible with the results found by Van der Swaelmen et al. (2016), which found a turnover in metallicity around $[\mathrm{Fe} / \mathrm{H}] \sim-0.6$ dex, indicating an enrichment 


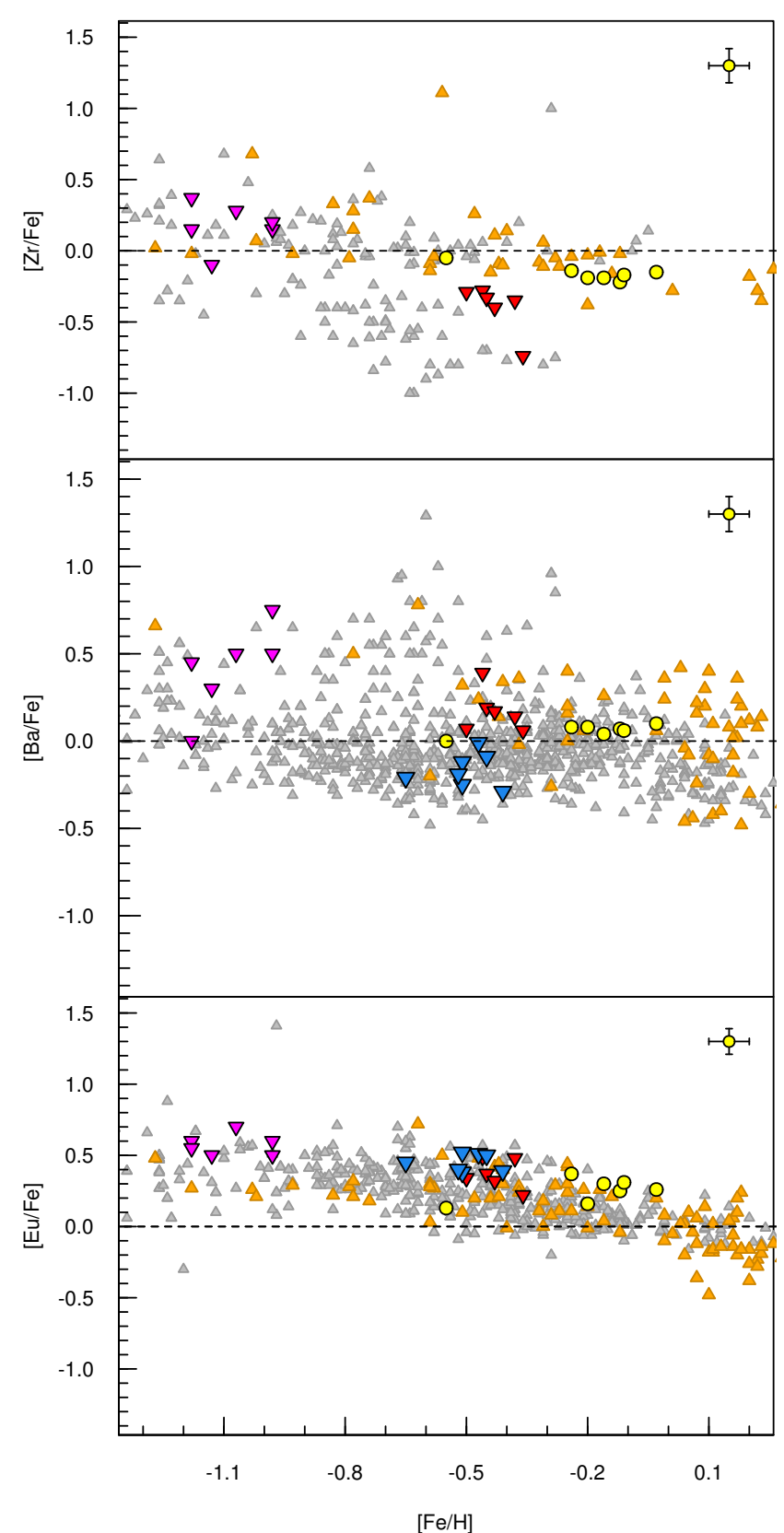

Fig. 11. $[\mathrm{Zr}, \mathrm{Ba}, \mathrm{Eu} / \mathrm{Fe}]$ vs. $[\mathrm{Fe} / \mathrm{H}]$. Filled yellow circles are our data for NGC 6528, filled blue triangles: NGC 6440 (Muñoz et al. 2017) filled red triangles: NGC 6441 (Gratton et al. 2006), filled magenta triangles: HP1 (Barbuy et al. 2016), filled orange triangles: bulge field stars (Van der Swaelmen et al. 2016), and filled gray triangles: Halo and disk stars (Fulbright 2000; François et al. 2007; Reddy et al. 2006; Barklem et al. 2005; Venn et al. 2004).

from SNe Ia. In this same context, Van der Swaelmen compared thick disk stars (Bensby et al. 2004) with a sample from bulge microlensed stars (Bensby et al. 2013); he found a small difference in the chemical enrichment between bulge and thick disk stars, apparently associated with different SN II/SN Ia ratios along time.

The decreasing of $\mathrm{Zr}$ with metallicity for the bulge field stars is evident in Fig. 11; this trend was detected by Johnson et al. (2012). Our sample of seven stars of NGC 6528 shows good agreement with the pattern of $\mathrm{Zr}$ in this regime of metallicity. Also, we found that our sample is depleted in $\mathrm{Zr}([\mathrm{Zr} / \mathrm{Fe}]=$ $-0.16)$.

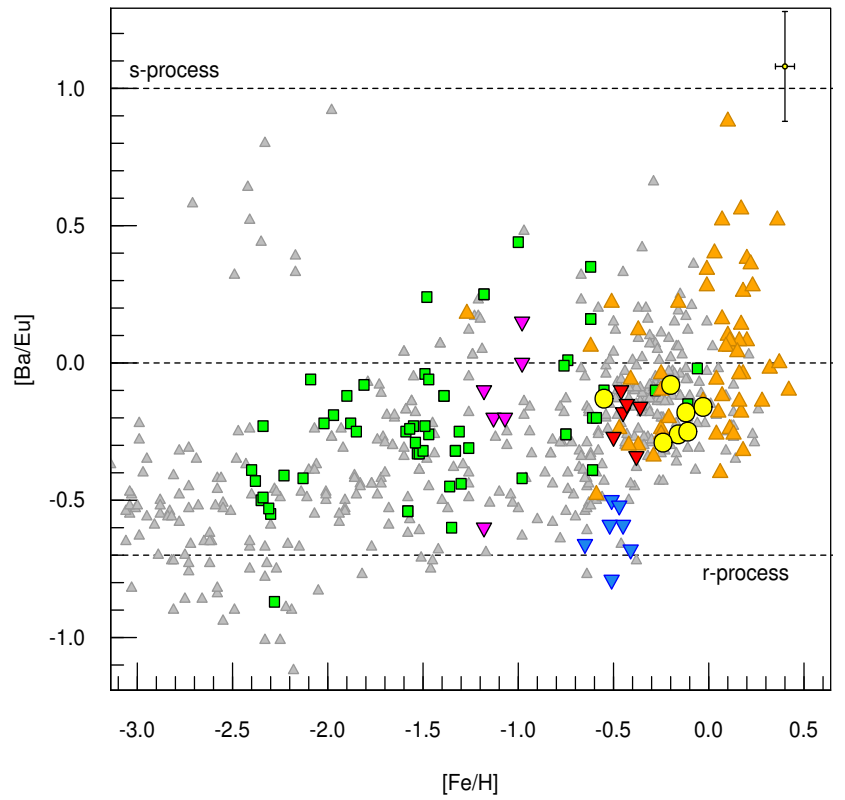

Fig. 12. $[\mathrm{Ba} / \mathrm{Eu}]$ vs. $[\mathrm{Fe} / \mathrm{H}]$. Filled yellow circles are our data for NGC 6528, filled blue triangles: NGC 6440 (Muñoz et al. 2017) filled red triangles: NGC 6441 (Gratton et al. 2006), filled magenta triangles: HP1 (Barbuy et al. 2016), filled orange triangles: bulge field stars (Van der Swaelmen et al. 2016), filled green square: GCs from Pritzl et al. (2005), and filled gray triangles: Halo and disk stars (Fulbright 2000; François et al. 2007; Reddy et al. 2006; Barklem et al. 2005; Venn et al. 2004).

\section{Conclusions}

This is the second paper of a series of papers about metal-rich bulge GCs. In this paper, we have presented a detailed chemical characterization of NGC 6528 from seven red giant members. Using VLT FLAMES/UVES high-resolution spectra, we measured 18 chemical elements and we performed a detailed error analysis. This allowed us to make a robust comparison with our first study on NGC 6440 as well as with many other Milky Way objects (bulge field stars, disk field stars, halo field stars, Galactic GCs). The most important results of this study are summarized in the following.

We found a candidate variable star (star \#5) in our sample. It turns out to be the most metal poor of the sample $([\mathrm{Fe} / \mathrm{H}]=$ $-0.55 \mathrm{dex}$ ), which may be due to its variability, but all its chemical patterns show good agreement with the other members of NGC 6528 . We obtained a mean iron abundance of $[\mathrm{Fe} / \mathrm{H}]=$ $-0.14 \pm 0.06$ dex in good agreement with previous literature values. We did not find a significant spread in iron, although our sample is small (after deleting the one dubious outlier).

We measure five iron-peak elements. These show very good agreement with the bulge field stars trend, especially with the bulge GC NGC 6553. The $\alpha$-elements also show good agreement with bulge field stars and NGC 6553. We did not find an extended $\mathrm{O}-\mathrm{Na}$ anticorrelation. This relationship is more vertical, similar to other bulge GCs, suggesting that only $\mathrm{Na}$ varies and $\mathrm{O}$ is fixed. We found no $\mathrm{Mg}$-Al anticorrelation. Neither did we find a real spread in either $\mathrm{Al}$ or $\mathrm{Mg}$. Heavy elements follow the trend of the bulge field stars and the bulge GCs. [Ba/Eu] versus $[\mathrm{Fe} / \mathrm{H}]$ shows good agreement with NGC 6441. Moreover, it is dominated by $r$-process material. The origin of NGC 6528 is undoubtedly similar to the bulge of the Milky Way, and especially to that of the bulge GC NGC 6553 . 

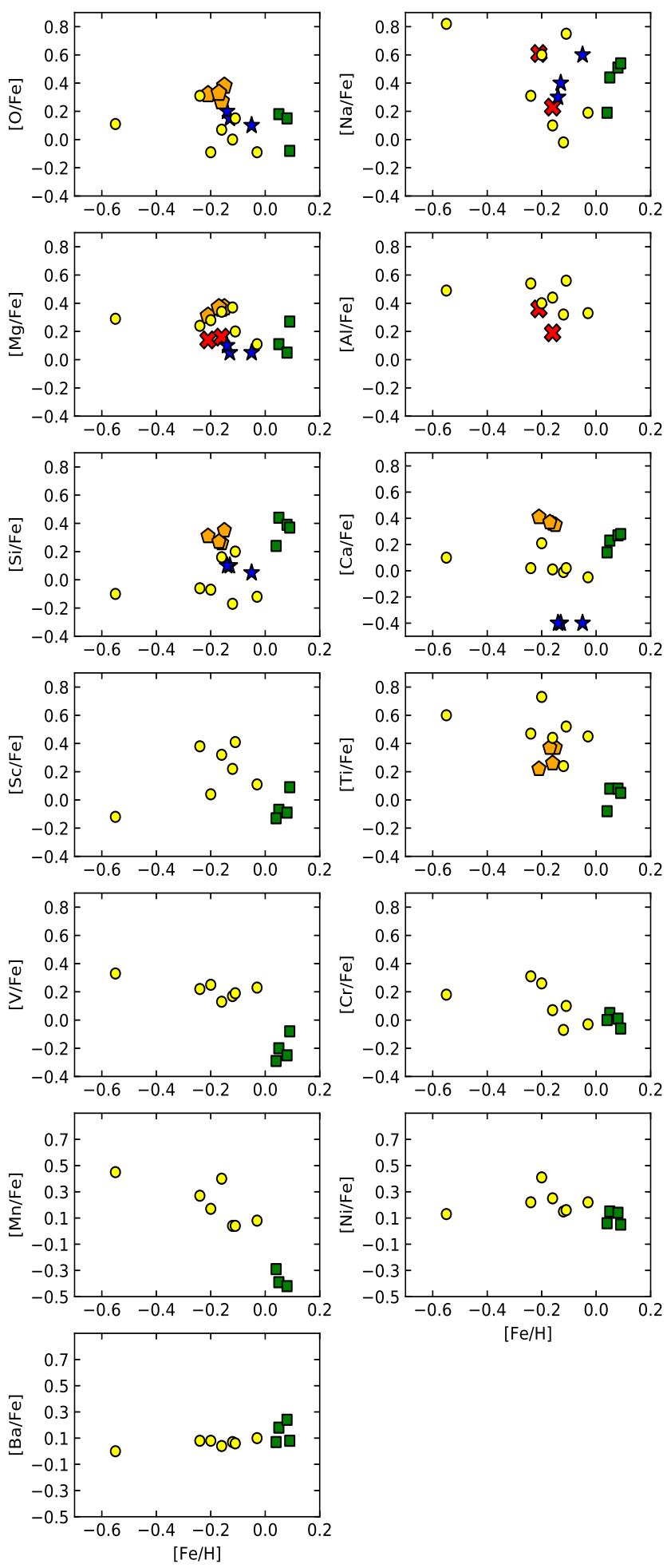

Fig. 13. Comparison of this study (yellow filled circles) with other studies for NGC 6528. Green filled squares from Carretta et al. (2001), orange filled pentagons from Origlia et al. (2005), filled blue stars from Zoccali et al. (2004), red filled crosses from Schiavon et al. (2017).

In all of the chemical comparisons we have made, NGC 6528 exhibits very good agreement with the general bulge chemical patterns, with the only minor exception being Mn. This behavior, together with its metallicity, could be indicative of a strong original contamination by $\mathrm{SN}$.
The $\alpha$-elements in the bulge field stars is a good indicator about the origin, because the bulge exhibits an overabundance in these elements (McWilliam \& Rich 1994; Zoccali et al. 2006). Our discovery for the $\alpha$-elements in NGC 6528 and for the majority of the bulge GCs analyzed in this study is compatible with this behavior.

Furthermore, we note that in the case of the O-Na anticorrelation, several of the bulge GCs show only variation in $\mathrm{Na}$, without a significant spread in O (Hp1, NGC 6440, NGC6528, NGC6553), again indicating a possible similar origin and evolution for these bulge GCs, and apparently different from that of Halo GCs. In particular, we highlight that for each chemical pattern in which we compared NGC 6528 with NGC 6553, we found very good agreement, indicating a similar evolution and origin.

According to Dinescu et al. (2003) the velocity of NGC 6528 is an indication of a radial orbit confined to the Galactic plane. Also, its rotation velocity shows good agreement with the velocity predicted for the Milky Way bar (Dinescu et al. 2003).

NGC 6528 is a GC with an age of 11 Gyr (Lagioia et al. 2014) and is one of the most metal-rich galactic GCs $([\mathrm{Fe} / \mathrm{H}]=$ 0.14). The chemical and dynamical patterns of NGC 6528 reveal this bulge GC to have a similar origin and evolution to the bulge of the Milky Way.

Finally, the most interesting of the above results is the lack of any $\mathrm{Na}-\mathrm{O}$ or $\mathrm{Mg}-\mathrm{Al}$ anticorrelation and the homogeneity of the $\mathrm{O}, \mathrm{Mg}$, and $\mathrm{Al}$ abundances, although the sample size is limited. This trend is in general in good agreement with other bulge GCs (NGC 6441, NGC 6553, NGC 6440). This could indicate a different evolution from that of the typical halo or disk GCs. As usual, additional studies, with much larger samples of clusters as well as more stars per cluster, will be needed to clearly define and understand these trends.

Acknowledgements. This work is based on observations collected at the European Organisation for Astronomical Research in the southern hemisphere under ESO programme ID 093.D-0286. We gratefully acknowledge use of data from ESO Public Survey programme ID 172.B-2002 taken with the VISTA telescope. We gratefully acknowledge support from the Chilean BASAL Centro de Excelencia en Astrofísica y Tecnologías Afines (CATA). S.V. gratefully acknowledges the support provided by FONDECYT reg. n. 1170518. C.C.C. is supported by CONICYT (Chile) throught Programa Nacional de Becas de Doctorado 2014 (CONICYT-PCHA/Doctorado Nacional/2014-21141084). D.G. also acknowledges financial support from the Dirección de Investigación y Desarrollo de la Universidad de La Serena through the Programa de Incentivo a la Investigación de Académicos (PIA-DIDULS). We would also like to thank the referee for his valuable comments and corrections.

\section{References}

Alonso-García, J., Dékány, I., Catelan, M., et al. 2015, AJ, 149, 99 Ballero, S. K., Matteucci, F., Origlia, L., \& Rich, R. M. 2007, A\&A, 467, 123 Barbuy, B., Hill, V., Zoccali, M., et al. 2013, A\&A, 559, A5 Barbuy, B., Cantelli, E., Vemado, A., et al. 2016, A\&A, 591, A53 Barklem, P. S., Christlieb, N., Beers, T. C., et al. 2005, A\&A, 439, 129 Bensby, T., Feltzing, S., \& Lundström, I. 2004, A\&A, 415, 155 Bensby, T., Yee, J. C., Feltzing, S., et al. 2013, A\&A, 549, A147

Blanton, M. R., Bershady, M. A., Abolfathi, B., et al. 2017, AJ, 154, 28 Carretta, E., Cohen, J. G., Gratton, R. G., \& Behr, B. B. 2001, AJ, 122, 1469 Carretta, E., Bragaglia, A., Gratton, R. G., et al. 2009a, A\&A, 505, 117 Carretta, E., Bragaglia, A., Gratton, R., \& Lucatello, S. 2009b, A\&A, 505, 139 Carretta, E., Bragaglia, A., Gratton, R. G., et al. 2010a, ApJ, 714, L7 Carretta, E., Bragaglia, A., Gratton, R. G., et al. 2010b, A\&A, 516, A55 Catelan, M., Minniti, D., \& Lucas, P. W. 2013, ArXiv e-prints [arXiv:1310.1996]

Cayrel, R., Depagne, E., Spite, M., et al. 2004, A\&A, 416, 1117

Cescutti, G., Matteucci, F., Lanfranchi, G. A., \& McWilliam, A. 2008, A\&A, 491, 401

Cescutti, G., Chiappini, C., \& Hirschi, R. 2018, IAU Symp., 334, 94

Cohen, R. E., Moni Bidin, C., Mauro, F., Bonatto, C., \& Geisler, D. 2017, MNRAS, 464, 1874 
Da Costa, G. S., Held, E. V., Saviane, I., \& Gullieuszik, M. 2009, ApJ, 705, 1481

D’Antona, F., Caloi, V., Montalbán, J., Ventura, P., \& Gratton, R. 2002, A\&A, 395, 69

D'Antona, F., Vesperini, E., D'Ercole, A., et al. 2016, MNRAS, 458, 2122

de Mink, S. E., Pols, O. R., Langer, N., \& Izzard, R. G. 2010, IAU Symp., 266, 169

Decressin, T., Meynet, G., Charbonnel, C., Prantzos, N., \& Ekström, S. 2007 , A\&A, 464, 1029

Dias, B., Barbuy, B., Saviane, I., et al. 2015, A\&A, 573, A13

Dias, B., Barbuy, B., Saviane, I., et al. 2016a, A\&A, 590, A9

Dias, B., Saviane, I., Barbuy, B., et al. 2016b, The Messenger, 165, 19

Dinescu, D. I., Girard, T. M., van Altena, W. F., \& López, C. E. 2003, AJ, 125 1373

Dotter, A., Chaboyer, B., Jevremović, D., et al. 2008, ApJS, 178, 89

François, P., Depagne, E., Hill, V., et al. 2007, A\&A, 476, 935

Fulbright, J. P. 2000, AJ, 120, 1841

Gallino, R., Arlandini, C., Busso, M., et al. 1998, ApJ, 497, 388

Gilmore, G., Randich, S., Asplund, M., et al. 2012, The Messenger, 147, 25

Gonzalez, O. A., Rejkuba, M., Zoccali, M., et al. 2012, A\&A, 543, A13

Gratton, R., Sneden, C., \& Carretta, E. 2004, ARA\&A, 42, 385

Gratton, R. G., Lucatello, S., Bragaglia, A., et al. 2006, A\&A, 455, 271

Gratton, R. G., Lucatello, S., Bragaglia, A., et al. 2007, A\&A, 464, 953

Gratton, R. G., Carretta, E., \& Bragaglia, A. 2012, A\&ARv, 20, 50

Harris, W. E. 1996, AJ, 112, 1487

Iwamoto, K., Brachwitz, F., Nomoto, K., et al. 1999, ApJS, 125, 439

Izzard, R. G., de Mink, S. E., Pols, O. R., et al. 2013, Mem. Soc. Astron. It., 84, 171

Johnson, C. I., Pilachowski, C. A., Simmerer, J., \& Schwenk, D. 2008, ApJ, 681, 1505

Johnson, C. I., Rich, R. M., Kobayashi, C., \& Fulbright, J. P. 2012, ApJ, 749, 175

Johnson, C. I., Rich, R. M., Kobayashi, C., Kunder, A., \& Koch, A. 2014, AJ, 148, 67

Kobayashi, C., Umeda, H., Nomoto, K., Tominaga, N., \& Ohkubo, T. 2006, ApJ, 653,1145

Krause, M., Charbonnel, C., Decressin, T., Meynet, G., \& Prantzos, N. 2013 A\&A, 552, A121

Lagioia, E. P., Milone, A. P., Stetson, P. B., et al. 2014, ApJ, 782, 50

Lecureur, A., Hill, V., Zoccali, M., et al. 2007, A\&A, 465, 799

Liu, C., Ruchti, G., Feltzing, S., \& Primas, F. 2017, A\&A, 601, A31

Marino, A. F., Villanova, S., Piotto, G., et al. 2008, A\&A, 490, 625

Marino, A. F., Milone, A. P., Piotto, G., et al. 2011a, ApJ, 731, 64

Marino, A. F., Sneden, C., Kraft, R. P., et al. 2011b, A\&A, 532, A8
Matteucci, F., Raiteri, C. M., Busson, M., Gallino, R., \& Gratton, R. 1993, A\&A, 272,421

Mauro, F., Moni Bidin, C., Geisler, D., et al. 2014, A\&A, 563, A76

McWilliam, A., \& Rich, R. M. 1994, ApJS, 91, 749

McWilliam, A., \& Smecker-Hane, T. A. 2005, ApJ, 622, L29

Mészáros, S., Martell, S. L., Shetrone, M., et al. 2015, AJ, 149, 153

Minniti, D. 1995, AJ, 109, 1663

Minniti, D., Lucas, P. W., Emerson, J. P., et al. 2010, New Astron., 15, 433

Mishenina, T. V., Kovtyukh, V. V., Soubiran, C., Travaglio, C., \& Busso, M. 2002, A\&A, 396, 189

Momany, Y., Ortolani, S., Held, E. V., et al. 2003, A\&A, 402, 607

Mucciarelli, A., Lapenna, E., Massari, D., Ferraro, F. R., \& Lanzoni, B. 2015, ApJ, 801, 69

Muñoz, C., Geisler, D., \& Villanova, S. 2013, MNRAS, 433, 2006

Muñoz, C., Villanova, S., Geisler, D., et al. 2017, A\&A, 605, A12

Mura-Guzmán, A., Villanova, S., Muñoz, C., \& Tang, B. 2018, MNRAS, 474, 4541

Origlia, L., \& Rich, R. M. 2004, AJ, 127, 3422

Origlia, L., Valenti, E., \& Rich, R. M. 2005, MNRAS, 356, 1276

Origlia, L., Rich, R. M., Ferraro, F. R., et al. 2011, ApJ, 726, L20

Origlia, L., Massari, D., Rich, R. M., et al. 2013, ApJ, 779, L5

Ortolani, S., Barbuy, B., Momany, Y., et al. 2011, ApJ, 737, 31

Pancino, E., Romano, D., Tang, B., et al. 2017, A\&A, 601, A112

Pritzl, B. J., Venn, K. A., \& Irwin, M. 2005, AJ, 130, 2140

Reddy, B. E., Tomkin, J., Lambert, D. L., \& Allende Prieto, C. 2003, MNRAS, 340,304

Reddy, B. E., Lambert, D. L., \& Allende Prieto, C. 2006, MNRAS, 367, 1329

Saito, R. K., Minniti, D., Dias, B., et al. 2012, A\&A, 544, A147

Saviane, I., da Costa, G. S., Held, E. V., et al. 2012, A\&A, 540, A27

Schiavon, R. P., Johnson, J. A., Frinchaboy, P. M., et al. 2017, MNRAS, 466, 1010

Shetrone, M., Venn, K. A., Tolstoy, E., et al. 2003, AJ, 125, 684

Simmerer, J., Ivans, I. I., Filler, D., et al. 2013, ApJ, 764, L7

Sneden, C. 1973, ApJ, 184, 839

Stellingwerf, R. F. 1978, ApJ, 224, 953

Straniero, O., Gallino, R., \& Cristallo, S. 2006, Nucl. Phys. A, 777, 311

Tang, B., Cohen, R. E., Geisler, D., et al. 2017, MNRAS, 465, 19

Van der Swaelmen, M., Barbuy, B., Hill, V., et al. 2016, A\&A, 586, A1

Venn, K. A., Irwin, M., Shetrone, M. D., et al. 2004, AJ, 128, 1177

Ventura, P., \& D’Antona, F. 2006, A\&A, 457, 995

Villanova, S., Geisler, D., Carraro, G., Moni Bidin, C., \& Muñoz, C. 2013, ApJ, 778,186

Zechmeister, M., \& Kürster, M. 2009, A\&A, 496, 577

Zoccali, M., Barbuy, B., Hill, V., et al. 2004, A\&A, 423, 507

Zoccali, M., Lecureur, A., Barbuy, B., et al. 2006, A\&A, 457, L1 